\title{
Metastability for the Ising Model on the Hypercube
}

\author{
Oliver Jovanovski ${ }^{1}$ (B)
}

Received: 6 August 2016 / Accepted: 27 January 2017 / Published online: 7 February 2017

(C) The Author(s) 2017. This article is published with open access at Springerlink.com

\begin{abstract}
We consider Glauber dynamics for the low-temperature, ferromagnetic Ising Model on the $n$-dimensional hypercube. We derive precise asymptotic results for the crossover time (the time it takes for the dynamics to go from the configuration with a " -1 " at every vertex, to the configuration with a " +1 " at each vertex) in the limit as the inverse temperature $\beta \rightarrow \infty$.
\end{abstract}

Keywords Metastability · Ising model · Interacting particle systems · Glauber dynamics · Hypercube

\section{Ising Model on the Hypercube}

Put in simple terms, metastability is the phenomenon describing a stochastic process that is temporarily trapped in the neighbourhood of a metastable state, away from the stable state which corresponds to the thermodynamic equilibrium. Usually this trap comes in the form of a local minimum of an associated energy function, and over a short time scale the observed process appears to be in a quasi-equilibrium. Viewed over a longer time scale, the process manages (after many unsuccessful attempts) to overcome the energy-barrier that separates it from a global minimum, which is often unique and the only true equilibrium.

In the physical world, observations of this phenomenon can be witnessed for example in magnetic hysteresis and in the condensation of an over-saturated vapour. In the context of statistical physics, the three main points of interest for metastability are the transition time from the metastable state to the stable state, the gate of critical configurations the process will visit in order to achieve the transition, and the tube of typical trajectories the system follows when making this transition. These have been studied intensively in the past decades, aided by the development of a number of powerful tools.

Oliver Jovanovski

o.jovanovski@math.leidenuniv.nl

1 Mathematical Institute, Leiden University, P.O. Box 9512, 2300 RA Leiden, The Netherlands 
The first of these tools is a method known as the pathwise approach and based on large deviation theory. Initially this was introduced by Cassandro et al. [11] for the Curie-Weiss model and the contact process on $\mathbb{Z}$, and later by Neves and Schonmann [21] for the Ising Glauber model on $\mathbb{Z}^{2}$. This approach was then generalized by Olivieri and Scoppola [22,23] and Catoni and Cerf [12] for reversible and non-reversible Markov chains. A particular strength of the pathwise approach is that it can be applied to study all three of the aforementioned points.

A second method, known as the potential-theoretic approach, was developed by Bovier et al. [6-9]. This method makes use of results from electrical network theory and their applications to reversible Markov chains, and was used by Bovier and Manzo [10], and Bovier et al. [5] to obtain sharper results for the Glauber and Kawasaki Ising model.

The pathwise and potential-theoretic approach are the subjects of two monographs: the first is treated by Olivieri and Vares in [24], the latter by Bovier and den Hollander in [4].

A third method, known as the martingale approach, was developed more recently by Beltran and Landim in [1-3] and applied to the study of metastability for the Ising model in $\mathbb{Z}^{2}$.

Since their development, these approaches have been used to study metastability for a variety of models. For instance, an analysis in $\mathbb{Z}^{d}$ for a nucleation-and-growth model was done by Dehghanpour and Schonmann [14], and for the $d$-dimensional Ising model by Cerf and Manzo [13]. Kotecky and Olivieri [18-20] also applied this method for the Glauber Ising model with isotropic, anisotropic and staggered interactions. More recently, a study of metastability for the Glauber Ising model on random graphs was done by Dommers [15] and Dommers et al. [16].

In this paper we use general theory from the potential theoretic approach to derive results for the Ising model set on an $n$-dimensional hypercube. This study is motivated by the distinct geometry of the hypercube. It is an expander graph, and should therefore give rise to very different dynamical behaviour (discussed in Sect. 1.4). At the same time, its strong symmetry makes it sufficiently tractable to fully exploit the potential theoretic approach in obtaining sharp results.

The general theory behind the potential-theoretic approach shows that in the limit $\beta \rightarrow \infty$ and under certain regularity conditions, the average crossover time-i.e. the time it takes for the process to go from the $\boxminus$ configuration (corresponding to a -1 spin at every vertex of the hypercube) to the $\boxplus$ configuration (corresponding to a +1 spin at every vertex of the hypercube)-behaves like $K \exp \left(-\beta \Gamma^{\star}\right)$ for some $K, \Gamma^{\star} \in \mathbb{R}^{+}$. We show that for the hypercube the required conditions are met (subject to necessary constraints on the parameters), obtain exact solutions for $K$ and $\Gamma^{\star}$, and give a full description of the critical configurations seen by the process when making the transition from $\boxminus$ to $\boxplus$. In order to do this, we investigate specific geometric properties of the hypercube and of the induced graph of the Markov process (explained further in Sect. 1.1). We will show that in case of the hypercube, $\Gamma^{\star}$ is proportional to the volume of the cube (i.e. it is of order $2^{n}$ ). This differs dramatically from the Ising model on a finite box in $\mathbb{Z}^{d}$, where $\Gamma^{\star}$ does not depend on the volume of the box (see [13] for instance).

\subsection{The Ising Model on the Hypercube}

We will denote the graph of the $n$-dimensional hypercube by $\mathcal{Q}_{n}=\left(V_{n}, E_{n}\right)$, where $V_{n}=$ $\{0,1\}^{n}$ are its vertices and $E_{n}=\left\{(v, w) \in V_{n} \times V_{n}:\|v-w\|_{1}=1\right\}$ its edges. Here for a vertex $v=\left(v_{1}, \ldots, v_{n}\right) \in V_{n}$, the norm $\|\cdot\|_{1}$ is defined by $\|v\|_{1}=\sum_{i=1}^{n} v_{i}$. If $\mathcal{Q}_{r}$ is an $r$-dimensional sub-cube of $\mathcal{Q}_{n}$ (a subgraph of size $2^{r}$ that is isomorphic to an $r$-dimensional 
hypercube, and hence all its vertices agree on $n-r$ co-ordinates), we shall (by a minor abuse of notation) write " $A \subseteq \mathcal{Q}_{r}$ " to mean that $A$ is a subset of the vertices in $\mathcal{Q}_{r}$. By "Ising Model on the hypercube" we are thinking of the configuration space $\Omega=\{+1,-1\}^{V_{n}}$ together with an associated Gibbs measure on this space, defined in (1.2). This configuration space corresponds to the assignment to each vertex of exactly one of two spins (either +1 or -1 ). Hence an equivalent representation of $\Omega$ is the power set $\mathcal{P}\left(V_{n}\right)$ of $V_{n}$, where $A \in \mathcal{P}\left(V_{n}\right)$ is identified with the configuration that assigns $(+1)$ to every vertex in $A$, and $(-1)$ to every vertex in $\bar{A}$ (the complement of $A$ ). Therefore we will (by further abuse of notation) identify $\Omega$ with $\mathcal{P}\left(V_{n}\right)$ and refer to the terms in $\mathcal{P}\left(V_{n}\right)$ (and hence $\Omega$ ) as configurations, whenever there is no threat of ambiguity.

Two special configurations (subsets) deserve their own symbols—we will denote by $\boxplus$ and $\boxminus$ the configurations $V_{n}$ and $\varnothing$ in $\Omega$ (equivalently, these are the two configurations with a $(+1) /(-1)$ assigned to every vertex). The Hamiltonian function $\mathcal{H}: \Omega \rightarrow \mathbb{R}$ associates an energy with each configuration $A \in \Omega$ according to

$$
\mathcal{H}(A)=-\frac{J}{2}\left(\left|E_{n}\right|-2|E(A, \bar{A})|\right)-\frac{h}{2}(|A|-|\bar{A}|)
$$

where for two subsets $U, W \subseteq V_{n}, E(U, W) \subseteq E_{n}$ is the set of all unoriented edges with one endpoint in $U$ and another in $W$. The parameters $J>0, h>0$ are fixed constants, known as the interaction and external field parameters, respectively. The Gibbs probability measure on $\Omega$ is given by

$$
\mu_{\beta}(A)=\frac{1}{Z_{n}} \exp (-\beta \mathcal{H}(A))
$$

with $\beta \geq 0$ being the inverse temperature and $Z_{n}$ the normalizing constant. Our interest is in the behaviour of the system when $\beta \rightarrow \infty$, thus we may take $J=1$, which simply corresponds to a rescaling of $\beta$ and $h$. Then with $J=1$, we will also assume throughout this paper that $0<h<n$, and some of our results will also require that $h$ is not an integer. The implications of, and reasons for having these assumptions will be discussed in Sects. 1.4 and 5 .

The final ingredient will be to define the dynamics on $\Omega$. To do this, let us first define

$$
\mathcal{E}_{n}=\left\{\left(A, A^{\prime}\right) \in \mathcal{P}\left(V_{n}\right) \times \mathcal{P}\left(V_{n}\right):\left|A \triangle A^{\prime}\right|=1\right\},
$$

where for two sets $A, A^{\prime} \subseteq V_{n}, A \triangle A^{\prime}=\left\{A \backslash A^{\prime}\right\} \cup\left\{A^{\prime} \backslash A\right\}$ denotes their symmetric difference. We consider continuous-time Glauber dynamics, which is a reversible, continuous-time Markov process $\left(\xi_{t}\right)_{t \geq 0}$ with (1.2) as its equilibrium measure, and is defined by the transition rates

$$
c_{\beta}\left(\xi, \xi^{\prime}\right)= \begin{cases}\exp \left(-\beta\left[\mathcal{H}\left(\xi^{\prime}\right)-\mathcal{H}(\xi)\right]_{+}\right), & \left(\xi, \xi^{\prime}\right) \in \mathcal{E}_{n} \\ 0 & \text { otherwise }\end{cases}
$$

where $\left[\mathcal{H}\left(\xi^{\prime}\right)-\mathcal{H}(\xi)\right]_{+}=\max \left\{0, \mathcal{H}\left(\xi^{\prime}\right)-\mathcal{H}(\xi)\right\}$. Thus, from (1.3) it is clear that $c_{\beta}$ defines single spin-flip dynamics, with transitions corresponding to a sign change at a single site.

\subsection{Metastability}

In order to discuss and describe metastable behaviour, we will need to investigate certain geometric quantities. The first of these is the communication height between two configurations $\xi, \xi^{\prime}$, defined by

$$
\Phi\left(\xi, \xi^{\prime}\right)=\min _{\gamma: \xi \rightarrow \xi^{\prime}} \max _{\sigma \in \gamma} \mathcal{H}(\sigma)
$$


where the minimum is taken over all paths $\gamma: \xi \rightarrow \xi^{\prime}$ on the graph $\left(\Omega, \mathcal{E}_{n}\right)$. The stability level of a configuration $\xi \in \Omega \backslash\{\boxplus\}$ is defined by

$$
\mathscr{V}_{\xi}=\min _{\zeta: \mathcal{H}(\zeta)<\mathcal{H}(\xi)} \Phi(\xi, \zeta)-\mathcal{H}(\xi)
$$

It is easy to see from the definition of $\mathcal{H}$ in (1.1) that the set of stable configurations,

$$
\Omega_{s}=\left\{\xi \in \Omega: \mathcal{H}(\xi)=\min _{\xi \in \Omega} \mathcal{H}(\xi)\right\}
$$

always reduces to $\Omega_{s}=\{\boxplus\}$. The set of metastable configurations is defined by

$$
\Omega_{m}=\left\{\xi \in \Omega \backslash\{\boxplus\}: \mathscr{V}_{\xi}=\max _{\xi \in \Omega \backslash\{\boxplus\}} \mathscr{V}_{\xi}\right\}
$$

and identifying this set is generally not a trivial task. We will show that for the Ising model on $\mathcal{Q}_{n}, \Omega_{m}=\{\boxminus\}$ whenever metastable behaviour occurs (see paragraph following Theorem 3). This justifies our next definition, namely the energy-barrier between the metastable and stable configurations,

$$
\Gamma^{\star}=\Phi(\boxminus, \boxplus)-\mathcal{H}(\boxminus) .
$$

Note from (1.1) that for any $\sigma \in \Omega$ (recall that we are taking $J=1$ ),

$$
\begin{aligned}
\mathcal{H}(\sigma)-\mathcal{H}(\boxminus)= & -\frac{J}{2}\left(\left|E_{n}\right|-2|E(\sigma, \bar{\sigma})|\right)-\frac{h}{2}(|\sigma|-|\bar{\sigma}|)+\frac{J}{2}\left(\left|E_{n}\right|-2|E(\boxminus, \boxplus)|\right) \\
& +\frac{h}{2}(|\boxminus|-n) \\
= & |E(\sigma, \bar{\sigma})|-h|\sigma|
\end{aligned}
$$

and hence

$$
\Gamma^{\star}=\min _{\gamma: \boxminus \rightarrow \boxplus} \max _{\sigma \in \gamma}(|E(\sigma, \bar{\sigma})|-h|\sigma|)
$$

We call paths $\gamma: \boxminus \rightarrow \boxplus$ that satisfy the minmax in (1.8) optimal paths.

One further point of interest will be the critical set $\mathscr{C}^{\star} \subseteq \Omega$ and the proto-critical set $\mathscr{P}^{\star} \subseteq \Omega$ of configurations, defined as the unique, maximal subset $\mathscr{C}^{\star} \times \mathscr{P}^{\star} \subseteq \Omega^{2}$ that satisfies the conditions

1. $\forall \xi \in \mathscr{P}^{\star}, \exists \xi^{\prime} \in \mathscr{C}^{\star}$ s.t. $\left(\xi, \xi^{\prime}\right) \in \mathcal{E}_{n}$ $\forall \xi \in \mathscr{C}^{\star}, \exists \xi^{\prime} \in \mathscr{P}^{\star}$ s.t. $\left(\xi, \xi^{\prime}\right) \in \mathcal{E}_{n}$

2. $\forall \xi \in \mathscr{P}^{\star}, \Phi(\xi, \boxminus)<\Phi(\xi, \boxplus)$

3. $\forall \xi \in \mathscr{C}^{\star}, \exists \gamma: \xi \rightarrow \boxplus$ s.t. $\max _{\zeta \in \gamma} \mathcal{H}(\zeta)-\mathcal{H}(\boxminus) \leq \Gamma^{\star}$

$$
\text { and } \gamma \cap\{\zeta \in \Omega: \Phi(\zeta, \boxminus)<\Phi(\zeta, \boxplus)\}=\emptyset
$$

Uniqueness follows from maximality and the observation that if $\left(\mathscr{C}_{1}^{\star}, \mathscr{P}_{1}^{\star}\right)$ and $\left(\mathscr{C}_{2}^{\star}, \mathscr{P}_{2}^{\star}\right)$ both satisfy the above conditions, then so does $\left(\mathscr{C}_{1}^{\star} \cup \mathscr{C}_{2}^{\star}, \mathscr{P}_{1}^{\star} \cup \mathscr{P}_{2}^{\star}\right)$. For any $\mathcal{A} \subseteq \Omega$, define

$$
\tau_{\mathcal{A}}=\inf \left\{t>0: \xi_{t} \in \mathcal{A}, \exists 0<s<t: \xi_{s} \neq \xi_{0}\right\}
$$

to be the first hitting time of the set $\mathcal{A} \subseteq \Omega$ once the starting configuration has been vacated. 
The following hypotheses are required in order to state the key theorems of the potential theoretic approach.

$$
\begin{array}{ll}
\text { (H1) } & \Omega_{m}=\{\boxminus\} \\
\text { (H2) } & \xi \rightarrow\left|\xi^{\prime} \in \mathscr{P}^{\star}:\left(\xi, \xi^{\prime}\right) \in \mathcal{E}_{n}\right| \text { is constant on } \mathscr{C}^{\star}
\end{array}
$$

Hypothesis (H1) is an essential hypothesis for all results given below. Indeed, the validity of (H1) will be verified in Theorem 3 where it is also shown that if (H1) is not satisfied, then the system does not display metastable behaviour. Hypothesis (H2) states that $\exists k \in \mathbb{N}$ such that every configuration $\xi \in \mathscr{C}^{\star}$ has exactly $k$ neighbours in $\mathscr{P}^{\star}$. This hypothesis is only necessary for the second result in Theorem 2 . We will verify the validity of (H2) in Sect. 4, where we also derive a description of the sets $\mathscr{P}^{\star}$ and $\mathscr{C}^{\star}$ defined in (1.9).

The potential-theoretic approach to metastability relates the crossover-time - the first hitting time of the configuration $\boxplus$ by a process starting at a metastable state- to the quantities defined above, via the following theorems.

Theorem 1 (Theorem 16.5 in [4]) Suppose that (H1) and (H2) hold. Then there exists a constant $K \in(0, \infty)$ such that

$$
\lim _{\beta \rightarrow \infty} \exp \left(-\beta \Gamma^{\star}\right) \mathbb{E}_{\boxminus}\left[\tau_{\boxplus}\right]=K .
$$

Theorem 2 (Theorem 16.4 in [4]) Suppose that (H1) and (H2) hold. Then

(a) $\lim _{\beta \rightarrow \infty} \mathbb{P}_{\boxminus}\left(\tau_{\mathscr{C}} \star<\tau_{\boxplus} \mid \tau_{\boxplus}<\tau_{\boxminus}\right)=1$.

(b) $\lim _{\beta \rightarrow \infty} \mathbb{P}_{\boxminus}\left(\tau_{\mathscr{C}} \star=\xi\right)=1 /\left|\mathscr{C}^{\star}\right|$ for all $\xi \in \mathscr{C}^{\star}$.

In order for these results to give substantial quantitative insight, one has to verify that (H1) and (H2) hold, and establish what $\Gamma^{\star}, K$ and $\mathscr{C}^{\star}$ amount to. This is the basis of our results.

\subsection{Results}

Our first result assures the validity of hypotheses (H1) and (H2).

Theorem 3 Suppose that $0<h<n$. Then the hypotheses (H1) and (H2) hold, and hence Theorem 1 and Theorem 2 apply.

It follows from the proof of Theorem 3 (given in Sect. 5) that the condition $h<n$ is essential. Indeed, for $h \geq n, \mathcal{H}$ has no local minima. That is, for every $\sigma \in \Omega$, there exists a finite path $\sigma=\sigma_{0}, \ldots, \sigma_{M}=\boxplus$ with $\left(\sigma_{i}, \sigma_{i+1}\right) \in \mathcal{E}_{n}$ and $\mathcal{H}\left(\sigma_{i}\right) \geq \mathcal{H}\left(\sigma_{i+1}\right), i=0, \ldots, M-1$, and thus no metastable behaviour is observed in this system (from (1.4) it follows that $c_{\beta}\left(\sigma_{i}, \sigma_{i+1}\right)=1$, and hence $\left.\lim _{\beta \rightarrow \infty} \mathbb{E}_{\boxminus}\left[\tau_{\boxplus}\right]<\infty\right)$.

Our second result gives a description of the set $\mathscr{C}^{\star}$. Recall that an isomorphism on $\mathcal{Q}_{n}$ is a bijection $\varphi: V_{n} \rightarrow V_{n}$ such that $(\varphi(v), \varphi(w)) \in E_{n}$ if and only if $(v, w) \in E_{n}$. For $A \subseteq V_{n}$, define

$$
\mathcal{M}(A)=\left\{A^{\prime} \subseteq V_{n}: A^{\prime}=\varphi(A) \text { for some isomorphism } \varphi\right\} .
$$

When $h<n-2$, define the sub-sets $\left\{W_{i}\right\}_{i=1}^{\lceil(n-h) / 2\rceil-1}$ of $V_{n}$ in the following way:

$$
\begin{aligned}
& W_{1}=\left\{v=\left(v_{1}, \ldots, v_{n}\right) \in V_{n}: v_{i}=0 \text { for } i \geq\lceil n-h-2\rceil\right\} \\
& W_{1}^{\prime}=\left\{v=\left(v_{1}, \ldots, v_{n}\right) \in V_{n}: v_{\lceil n-h-2\rceil}=1, v_{i}=0 \text { for } i>\lceil n-h-2\rceil\right\}
\end{aligned}
$$




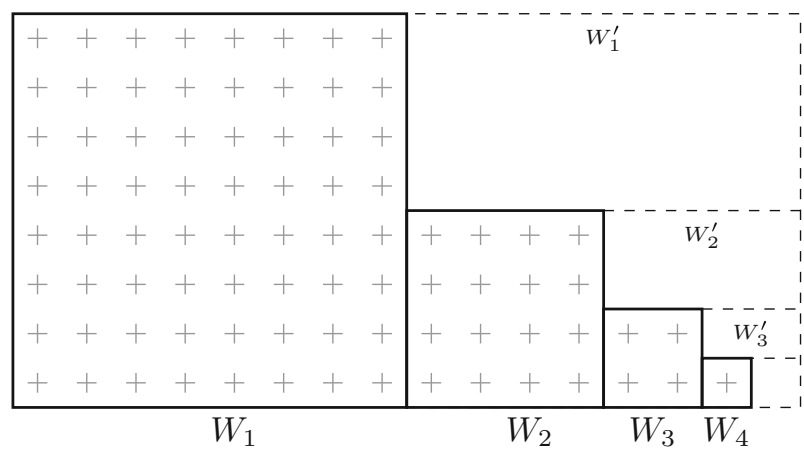

Fig. 1 Schematic representation of the critical configuration $\bigcup_{i=1}^{\lceil(n-h) / 2\rceil} W_{i} \in \mathscr{C}^{\star}$. In this example, $\lceil(n-h) / 2\rceil=4$. Note that $\left|W_{3}\right|=4$, which is the case if and only if $\lceil n-h\rceil$ is even

and for $2 \leq j \leq\lceil(n-h) / 2\rceil$

$$
\begin{aligned}
& W_{j}=\left\{v \in W_{j-1}^{\prime}: v_{i}=0 \text { for } i=\lceil n-h-2 j\rceil,\lceil n-h-2 j+1\rceil\right\} \\
& W_{j}^{\prime}=\left\{v \in W_{j-1}^{\prime}: v_{\lceil n-h-2 j+1\rceil}=0, v_{\lceil n-h-2 j\rceil}=1\right\} .
\end{aligned}
$$

Lastly, let $W_{\lceil(n-h) / 2\rceil}$ be the set with the single vertex in $w \in W_{\lceil(n-h) / 2\rceil-1}^{\prime}$ that satisfies $w_{i}=$ 0 for all $1 \leq i \leq\lceil n-h-2(\lceil(n-h) / 2\rceil-1)-1\rceil$. Hence for $1 \leq i \leq\lceil(n-h) / 2\rceil-1$, $W_{i}$ is the set of vertices of a $\lceil n-h-2\rceil$-dim sub-cube of $\mathcal{Q}_{n}$, and for $i>1$ the set $W_{i}$ is adjacent to all of $W_{1}, \ldots, W_{i-1}$. By this we mean that for every $1 \leq j<i \leq\lceil(n-h) / 2\rceil$, $W_{j} \cap W_{i}=\emptyset$ and $\forall w \in W_{i}, \exists v \in W_{j}$ such that $(w, v) \in E_{n}$. See Figure 1 above.

Theorem 4 Suppose that $h<n$ and $h$ is not integer valued. If $n-2 \leq h<n$, then $\mathscr{C}^{\star}$ is the set of all singleton configurations-i.e. $\mathscr{C}^{\star}$ is the set of all configurations that have exactly one vertex with $a+1$ spin. If $0<h<n-2, \mathscr{C}^{\star}=\mathcal{M}\left(\bigcup_{i=1}^{\lceil(n-h) / 2\rceil} W_{i}\right)$.

Thus for $h<n-2$, critical configurations take the shape of a series of shrinking, adjacent sub-cubes of $\mathcal{Q}_{n}$ (with the smallest being 1-dimensional if $\lceil n-h\rceil$ is odd and 2-dimensional otherwise) together with a protuberance (in the form of a single vertex) which is adjacent to all the other sub-cubes.

Our third result determines the value of the energy-barrier $\Gamma^{\star}$ in terms of the parameters of the system.

Theorem 5 Suppose that $h<n$. Then the energy-barrier $\Gamma^{\star}$ is given by

$$
\Gamma^{\star}=\frac{1}{3}(2-h+\lfloor h\rfloor)\left(2^{\lceil n-h\rceil}+2 \epsilon-1\right)-\epsilon
$$

where $\epsilon=\lceil n-h\rceil \bmod 2$.

Our fourth result gives the prefactor $K$ under the assumption that $h$ is not an integer.

Theorem 6 Suppose that $0<h<n$ and $h$ is not integer-valued. Then

$$
K=2^{-n}\left(1+\frac{1}{n}\right)
$$


for $h \geq n-2$, and

$$
K=\frac{3\lceil h\rceil !}{(1+\epsilon) n ! 2^{n}}
$$

for $0<h<n-2$, where $\epsilon=\lceil n-h\rceil \bmod 2$.

\subsection{Discussion}

From the perspective of the potential-theoretic approach to metastability, the theorems in Sect. 1.3 give a complete description of the metastable behaviour of Ising spin-flip dynamics on the $n$-dimensional hypercube. The metastable regime is characterized by the inequality $0<h<n$. Within this regime, and for a fixed value of $h$, we see from (1.14) that

$$
\Gamma^{\star} \approx \frac{1}{3} 2^{-\lfloor h\rfloor}(2-h+\lfloor h\rfloor) 2^{n},
$$

showing that $\Gamma^{\star}$ grows proportionally to the volume of $V_{n}$. A look at equation (1.8) hints that this should indeed be the case. The hypercube $\mathcal{Q}_{n}$ is an expander graph (which can be easily concluded from Theorem 7), meaning that there is some $\rho>0$ such that

$$
\liminf _{n \rightarrow \infty} \min _{A \subseteq V_{n}, 1 \leq|A| \leq\left|V_{n}\right| / 2} \frac{|E(A, \bar{A})|}{|A|} \geq \rho .
$$

Thus from (1.8) it is clear that for any such graph, $\Gamma^{\star} \geq(\rho-h) \frac{\left|V_{n}\right|}{2}$, growing linearly (or faster) with the volume of the graph whenever $h$ is sufficiently small.

Critical configurations take the shape of a series of adjacent cubes that are decreasing in size, with the final cube being a single vertex - a protuberance, similar to what is also observed for the Glauber Ising model on $\mathbb{Z}^{2}$. A noteworthy distinction from the $\mathbb{Z}^{d}$ case is that on $\mathcal{Q}_{n}$, adjacent cubes decrease in dimension arithmetically by 2 , whereas in $\mathbb{Z}^{d}$ the critical configuration is a union of 'quasi-squares' that decrease in dimension arithmetically by $1\left([13,14]\right.$ give a description of critical droplets in $\left.\mathbb{Z}^{d}\right)$.

Due to the large size of $\mathscr{C}^{\star}$, the prefactor $K$ decays in a super-polynomial way with respect to the volume of $V_{n}$ :

$$
K \approx\left|V_{n}\right|^{-\left(1+\log \log a\left|V_{n}\right|\right)}
$$

for some $a>0$. In contrast, for the Glauber Ising model on $\mathbb{Z}^{2}$ (where the Ising model is set on an $n \times n$ torus, denoted by $\Lambda_{n}$ ), it is known that $K=c\left|\Lambda_{n}\right|^{-1}$ for some $c>0$ (see Theorem 17.4 in [4]). Indeed, in $\mathbb{Z}^{2}$ a critical configuration can be described as a quasi-square-an $\ell_{1} \times \ell_{2}$ rectangle with $\ell_{1}, \ell_{2} \in \mathbb{N},\left|\ell_{1}-\ell_{2}\right|-1$. Hence in that case $\mathscr{C}^{\star}$ is obtained by taking all lateral translations of two quasi-squares (one is $\ell_{1} \times \ell_{2}$, and the other $\ell_{2} \times \ell_{1}$ ). Clearly this is a proportion of the area $\left|\Lambda_{n}\right|$. The hypercube permits a much larger set of isomorphic translations, resulting in $\mathscr{C}^{\star}$ being a considerably bigger set.

From the proof of Theorem 5 and Lemma 5 it is evident that when $h$ is an integer, optimal paths can contain a 'plateau' at the top. This has no implication on $\Gamma^{\star}$, but it does complicate the description of the set $\mathscr{C}^{\star}$, and would require a separate (and somewhat more difficult) analysis to determine the prefactor $K$.

\subsection{Outline of the Paper}

Our main focus will be on particular geometric properties of the hypercube that relate to the triplet $\left(\Gamma^{\star}, \mathscr{C}^{\star}, K\right)$. In Sect. 2 we will first establish some known results related to isoperi- 
metric inequalities on the hypercube, followed by a new result on this subject (Lemma 1). These results are applied in Sect. 3 to isolate local and global maxima of the energy function $\mathcal{H}$ given in (1.1) along an optimal path, and to prove Theorem 4, Theorem 5 and the validity of hypothesis (H2) in Theorem 3. In Sect. 4 we prove Theorem 6 by computing the prefactor $K$. In Sect. 5 we give a proof of hypothesis (H1) in Theorem 3. Sect. 6 contains the proof of Lemma 1, which together with Theorem 7 gives a description of all sets that solve the isoperimetric problem in equation (2.2).

\section{Isoperimetric Inequalities for the Hypercube}

In this section we state an edge-isoperimetric problem and a result from [17] that gives a solution to this problem. We show in Lemma 1 that this solution is in fact the only one, up to $\mathcal{M}$-equivalence (as defined in (1.11)). We use this to define an optimal path $\gamma: \boxminus \rightarrow \boxplus$ in Lemma 2.

The definitions (1.1) and (1.7) suggest that $\Gamma^{\star}$ will be closely related to the following edge-isoperimetric problem: given a graph $G=(V, E)$ and integer $1 \leq k \leq|V|$, what is $\min \{|E(A, \bar{A})|: A \subseteq V,|A|=k\}$ ? We will say a set $A$ has minimal edge-boundary if it satisfies this minimum. Solutions to this problem are known for the graph $\mathcal{Q}_{n}$ (see [17]). Aside from determining the minimum above, we will also need to identify all subsets of $V_{n}$ that have a minimal edge-boundary.

Define the function $q: \mathbb{N}_{0} \rightarrow \mathbb{N}_{0}$ as follows. For $i \in \mathbb{N}_{0}$, let $i=\sum_{j=1}^{\infty} a_{j} 2^{j-1}$ be the binary decomposition of $i$, and let $q(i)=\sum_{i=1}^{\infty} a_{i}$. The following gives a solution to the edge-isoperimetric problem for the hypercube.

Theorem 7 (Theorem 1.4 and 1.5 in [17]) For $k \in \mathbb{N}, 1 \leq k \leq 2^{n}$, define $\gamma_{k} \subseteq V_{n}$ by

$$
\gamma_{k}=\left\{v=\left(v_{1}, \ldots, v_{n}\right) \in V_{n} \mid \sum_{i=1}^{n} v_{i} 2^{i-1}<k\right\}
$$

and note that $\left|\gamma_{k}\right|=k$. Then

$$
\left|E\left(\gamma_{k}, \overline{\gamma_{k}}\right)\right|=\min _{S \subseteq V_{n},|S|=k}|E(S, \bar{S})|=n k-2 \sum_{i=1}^{k-1} q(i)
$$

For a set $S$ of size $k$, we will say that $|E(S, \bar{S})|$ is minimal if $S$ has a minimal edgeboundary.

Definition 1 (See also good set in [17]) A set $S \subseteq V_{n}$ with $|S|=k$ is called a good set if

(1) $k=1$, or

(2) if $2^{r}<k \leq 2^{r+1}$ for some $0 \leq r \leq n-1$ and there is some $r+1$ dimensional sub-cube $C_{r+1}$ containing $S$, such that $C_{r+1}$ is the union of two disjoint $r$-dimensional sub-cubes, $C_{r+1}=\left(C_{r}^{1}, C_{r}^{2}\right)$ with respective vertex sets $V_{r}^{1}$ and $V_{r}^{2}$, which satisfy $\left|S \cap V_{r}^{1}\right|=2^{r}$ and $S \cap V_{r}^{2}$ is a good set.

In other words, $S$ is a good set if it 'decomposes' into a disjoint union of adjacent sub-cubes that decrease in size. It is shown in [17] that if $S$ is a good set, then $|E(S, \bar{S})|$ is minimal. Equivalently, every good set $S$ makes $|E(S, S)|$ maximal (i.e. for any $U \subseteq V_{n}$ of size $|S|$, $|E(S, S)| \geq|E(U, U)|)$. It is easy to verify that (2.1) defines a good set for every $k$, and 


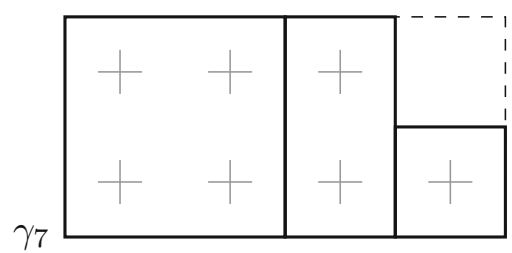

Fig. 2 From the definition of $\gamma_{k}$ in (2.1) it follows that the binary decomposition of $k$ completely describes the configuration $\gamma_{k}$. For example, $k=7=2^{0}+2^{1}+2^{2}$ implies that $\gamma_{7}$ is a configuration consisting of a 2-dim, a 1-dim, and a 0-dim sub-cube, and there are edges between any two of these three sub-cubes

thus by symmetry, the set of all good sets is given by $\mathcal{M}\left(\gamma_{k}\right)$. Hence every $\xi \in \mathcal{M}\left(\gamma_{k}\right)$ has minimal edge-boundary. See Figure 2 above.

This information will be sufficient to compute $\Gamma^{\star}$. However, in order to determine the prefactor $K$ in Theorem 1, we will see that it is necessary to examine all sets of a particular volume that have minimal edge-boundary. The following lemma proves that the sets $\left\{\gamma_{k}\right\}_{k=1}^{2^{n}}$ in Theorem 7 and their isomorphic translations are the only sets with minimal edge-boundary. To the best of the author's knowledge, this result has not been established in any previous work.

Lemma 1 Let $S \subseteq V_{n}$ be a subset of the hypercube. Then $|E(S, \bar{S})|$ is minimal if and only if $S \in \mathcal{M}\left(\gamma_{|S|}\right)$. Equivalently, $|E(S, \bar{S})|$ is minimal if and only if $S$ is a good set.

The proof of Lemma 1 is given in Sect. 6 .

Let $\gamma_{0}=\boxminus$, and note that the path $\gamma: \boxminus \rightarrow \boxplus$ given by

$$
\gamma=\left(\gamma_{0}, \gamma_{1}, \ldots, \gamma_{2^{n}-1}, \boxplus\right)
$$

as defined in (2.1) is a Glauber path (i.e. a path in $\left(\Omega, \mathcal{E}_{n}\right)$ ), since by definition the set $\gamma_{k+1}=$ $\gamma_{k} \cup\{w\}$ where $w=\left(w_{1}, \ldots, w_{n}\right) \in V_{n}$ is the unique vertex that satisfies $\sum_{i=1}^{n} w_{i} 2^{i-1}=$ $k+1$. By Theorem 7 we have the following immediate conclusion.

Lemma 2 The path $\gamma$ in (2.3) is a uniformly optimal path. That is, for all $\sigma \in \Omega, \mathcal{H}(\sigma) \geq$ $\mathcal{H}\left(\gamma_{|\sigma|}\right)$.

\section{Critical Configurations and Computation of the Energy-Barrier $\Gamma^{\star}$}

From Lemma 2 we know that the path $\gamma$ in (2.3) is an optimal path. In this section we prove Theorem 5 by computing the maximum value $\mathcal{H}$ attains along this path. The proof yields the volume of the first configuration along $\gamma$ that attains the value $\Gamma^{\star}$, which we use to prove Theorem 4 . We end the section with a brief argument justifying hypothesis (H2) in Theorem 3.

We begin with the following elementary result.

Lemma 3 For any $0 \leq r \leq n$,

$$
\sum_{i=1}^{2^{r}-1} q(i)=r 2^{r-1}
$$


Proof The proof is by induction. Note that (3.1) is clearly true for $r \in\{0,1\}$. Suppose that this also holds for all $r \in\{1, \ldots, k\}$. Then

$$
\sum_{i=1}^{2^{k+1}-1} q(i)=\sum_{i=1}^{2^{k}-1} q(i)+\sum_{i=2^{k}}^{2^{k+1}-1} q(i)=k 2^{k-1}+2^{k}+\sum_{i=0}^{2^{k}-1} q(i)=(k+1) 2^{k}
$$

The second equality follows from the observation that for any $0 \leq i<2^{k}$, the binary expansion of the number $2^{k}+i$ has exactly one more " 1 " than the binary expansion of the number $i$. Note that the right-most term in (3.2) is equivalent to (3.1) with $r=k+1$. This completes the proof.

A different proof of Lemma 3 is also given in [17].

Lemma 4 Let $1 \leq j<n-1$ and $1 \leq a<2^{n}$, and let the binary expansion of a be given by $a=\sum_{i=1}^{n} a_{i} 2^{i-1}, a_{i} \in\{0,1\}$. Suppose also that $a_{j}=1$ and $a_{j+1}=0$, and let $b=a+2^{j-1}$. Then

$$
\sum_{i=1}^{b-1} q(i)=\sum_{i=1}^{a-1} q(i)+\left(j+1+2 \sum_{i=j+2}^{n} a_{i}\right) 2^{j-2}
$$

Proof Observe first that the binary expansion of $b$ is obtained from the binary expansion of $a$ by switching $a_{j}$ with $a_{j+1}$. Suppose first that $a<2^{j}$, so that $a_{j}$ is the last " 1 " appearing in the binary expansion of $a$. Then $a=2^{j-1}+c$ for some $c<2^{j-1}$ and from Lemma 3 it follows that

$$
\sum_{i=1}^{a-1} q(i)=\sum_{i=1}^{2^{j-1}-1} q(i)+\sum_{i=2^{j-1}}^{2^{j-1}+c-1} q(i)=(j-1) 2^{j-2}+c+\sum_{i=0}^{c-1} q(i)
$$

while

$$
\sum_{i=1}^{b-1} q(i)=j 2^{j-1}+c+\sum_{i=1}^{c-1} q(i)=\sum_{i=1}^{a-1} q(i)+(j+1) 2^{j-2}
$$

which agrees with (3.3). Now suppose that $a \geq 2^{j}$, and hence $a \geq 2^{j+1}$ since $a_{j+1}=0$. Let $\tilde{a}=\sum_{i=1}^{j} a_{i} 2^{i-1}$ and $\tilde{b}=\tilde{a}+2^{j-1}$, and note that for every $\tilde{a} \leq \tilde{s}<\tilde{b}$ and $s=$ $\tilde{s}+\sum_{i=j+2}^{n} a_{i} 2^{i-1}$ we have

$$
q(s)=q(\tilde{s})+\sum_{i=j+2}^{n} a_{i} .
$$

Hence it follows from (3.4) that

$$
\sum_{i=a}^{b-1} q(i)=\sum_{i=\tilde{a}}^{\tilde{b}-1} q(i)+(b-a)\left(\sum_{i=j+2}^{n} a_{i}\right)=\left(j+1+2 \sum_{i=j+2}^{n} a_{i}\right) 2^{j-2}
$$

which proves (3.3).

We can now proceed with proving Theorem 5.

Proof of Theorem 5 Recall from (2.3) the definition of the path $\gamma: \boxminus \rightarrow \boxplus$. For $0 \leq k \leq 2^{n}$, define

$$
g(k)=\left|E\left(\gamma_{k}, \overline{\gamma_{k}}\right)\right|-h k .
$$


Then from (1.8) and Lemma 2 it follows that

$$
\begin{aligned}
\Gamma^{\star} & =\max _{0 \leq k \leq 2^{n}} g(k) \\
& =\max _{0 \leq k \leq 2^{n}}\left\{k(n-h)-2 \sum_{i=1}^{k-1} q(i)\right\}
\end{aligned}
$$

We are interested in finding any $k \in\left\{0, \ldots, 2^{n}\right\}$ such that $g(k)=\Gamma^{\star}$, and then computing $g(k)$. Uniqueness of $k$ will follow whenever $h$ is not integer valued. In general (i.e. when $h \in \mathbb{N}$ is permitted), $g$ may attain its maximum at more than one place, and therefore in the constructive proof below we will refer to a particular global maximum as our global maximum.

We will first show that if $k$ is any local (or global) maximum of $g$, it must have exactly $\delta=\lceil(n-h) / 2\rceil$ digits equal to "1" in its binary decomposition. Starting with any such local maximum $k=\sum_{i=0}^{n-1} k_{i} 2^{i-1}$, we will show that if $\max \left\{i: k_{i}=1\right\} \neq\lceil n-h-1\rceil$, we can "shift" the " 1 "' 's in the decomposition of $k$ (as was done in Lemma 4) to obtain a different local maximum $k^{\prime}$ that satisfies $\max \left\{i: k_{i}^{\prime}=1\right\}=\lceil n-h-1\rceil$ and $g(k) \leq g\left(k^{\prime}\right)$ (see Figure 3). This will determine where the final " 1 " in the decomposition of our global maximum should be. The same argument will also show where the other $\delta-1$ " 1 "s in the binary decomposition of our global maximum should be. We will thus obtain a $k$ that attains the maximum in (3.6).

The function $g$ is decreasing on $\{k, k+1\}$ if and only if $g(k+1) \leq g(k)$, which is equivalent to

$$
2\left(\sum_{i=1}^{k} q(i)-\sum_{i=1}^{k-1} q(i)\right)=2 q(k) \geq(n-h)
$$

Similarly, $g$ is increasing on $\{k-1, k\}$ if and only if $2 q(k-1) \leq(n-h)$. Observe that $q(k)-q(k-1) \leq 1$, hence local maxima of $g$ occur at values $k$ that have exactly $\delta=$ $\lceil(n-h) / 2\rceil$ digits equal to "1" in their binary expansion, while $k-1$ has at most $\delta$ digits equal to " 1 " in its binary expansion. Observe also that if $k \geq 2$ is even, then $q(k) \leq q(k-1)$, while if $k \geq 3$ is odd, $q(k)=q(k-1)+1$. Hence, in order to find a global maximum it suffices to only consider odd $k$, with $k-1$ having exactly $\delta-1$ digits equal to " 1 " in its binary expansion.

Now suppose that $k^{(1)}$ is an integer that satisfies the above conditions, with its binary expansion given by $k^{(1)}=\sum_{i=1}^{n} k_{i}^{(1)} 2^{i-1}$. Furthermore, suppose that $k_{j}^{(1)}=1$ and $k_{j+1}^{(1)}=0$ for some $j \geq 1$. Let $k^{(2)}=k^{(1)}+2^{j-1}$, so that the binary expansion of $k^{(2)}$ is obtained from that of $k^{(1)}$ by switching $k_{j}^{(1)}$ with $k_{j+1}^{(1)}$. By Lemma 4 we have that

$$
\begin{aligned}
g\left(k^{(2)}\right)-g\left(k^{(1)}\right) & =\left(k^{(2)}-k^{(1)}\right)(n-h)-2\left(\sum_{i=1}^{k^{(2)}-1} q(i)-\sum_{i=1}^{k^{(1)}-1} q(i)\right) \\
& =2^{j-1}\left(n-h-j-1-2 \sum_{i=j+2}^{n} k_{i}^{(1)}\right)
\end{aligned}
$$

We can now use (3.8) to compare the local maxima of $g$ in order to find its global maximum. Starting with any $k=\sum_{i=1}^{n} k_{i} 2^{i-1}$ that satisfies the aforementioned conditions ( $k$ is odd, $k$ has $\delta$ digits equal to " 1 " in its binary expansion, $k-1$ has $\delta-1$ digits equal to " 1 " in its 

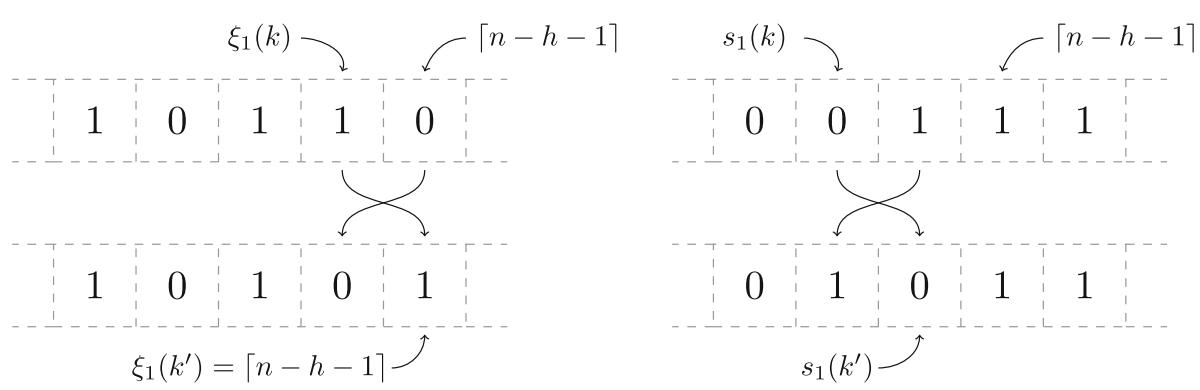

Fig. 3 In the top-left diagram, $\xi_{1}(k)<\lceil n-h-1\rceil$. The bottom-left diagram is the outcome of a 'switch' that corrects this, resulting in $k^{\prime}$ with $g\left(k^{\prime}\right) \geq g(k)$. In the top-right diagram, $\xi_{1}(k)>\lceil n-h-1\rceil$. To correct this, the first step is for the " 0 " at $s_{1}(k)$ to be switched with the " 1 " at $s_{1}(k)+1$, yielding $k^{\prime}$ with $s_{1}\left(k^{\prime}\right)=s_{1}(k)+1$ and $g\left(k^{\prime}\right) \geq g(k)$

binary expansion), let $\xi_{1}(k)=\max \left\{i: k_{i}=1\right\}$. We will show that if $k$ is a global maximum, $\xi_{1}(k)=\lceil n-h-1\rceil$ or $\xi_{1}(k)=1$ if $\lceil n-h-1\rceil=0$.

If $\xi_{1}(k)<n-h-1$, then by (3.8) we can switch the values of $k_{\xi_{1}(k)}(=1)$ and $k_{\xi_{1}(k)+1}$ $(=0)$ to obtain a local maximum $k^{\prime}$ such that $g(k)<g\left(k^{\prime}\right)$. We can repeat this 'switch' procedure until the final " 1 " is the $\lceil n-h-1\rceil^{\text {th }}$ term. With every switch we observe a new local maximum of $g$ that is greater than all previously observed ones (see Remark 1 below for the case $\lceil n-h-1\rceil=0)$.

We want to show that if $\xi_{1}(k) \geq\lceil n-h-1\rceil+1$, we can apply Lemma 4 again to shift the final " 1 " one space 'back' and obtain some $k *$ with $\xi_{1}(k *)=\xi_{1}(k)-1$ and $g(k *) \geq g(k)$. In order to do this, we need that $k_{\xi_{1}(k)}=0$, which may not be immediately the case. Hence we take the nearest " 0 " preceding $\xi_{1}(k)$ and shift it 'forward' until we have a value $k *$ with $k * \xi_{1}(k)=0$. See right-hand illustration in Figure 3 .

Formally, this is done as follows. Let $s_{1}(k)=\max \left\{i<\xi_{1}(k): k_{i}=0\right\}$ and let $k^{\prime}$ be the result of switching the terms $k_{s_{1(k)}}(=0)$ and $k_{s_{1}(k)+1}(=1)$ in the binary expansion of $k$. Then again from (3.8) it follows that

$$
\begin{aligned}
g\left(k^{\prime}\right)-g(k) & =-2^{s_{1}(k)-1}\left(n-h-s_{1}(k)-1-2 \sum_{i=s_{1}(k)+2}^{n} k_{i}\right) \\
& =-2^{s_{1}(k)-1}\left(n-h-s_{1}(k)-1-2\left(\xi_{1}(k)-s_{1}(k)-1\right)\right) \\
& =2^{s_{1}(k)-1}\left(2 \xi_{1}(k)-(n-h-1)-s_{1}(k)-2\right) \geq 0
\end{aligned}
$$

Thus by switching the values of $k_{s_{1}(k)}$ and $k_{s_{1}(k)+1}$, we obtain a local maximum $k^{\prime}$ which satisfies $g\left(k^{\prime}\right)>g(k)$. We continue in a recursive manner. Let $v_{0}=k^{\prime}$, and let $v_{i+1}$ be obtained from $v_{i}$ by switching the " 0 " at $s_{1}\left(v_{i}\right)$ with the " 1 " at $s_{1}\left(v_{i}\right)+1$ in the binary decomposition of $v_{i}$. Let $M=\min \left\{i: \xi_{1}\left(v_{i}\right)=\lceil n-h-1\rceil\right\}$. It is easy to check that $g\left(v_{0}\right) \leq \ldots g\left(v_{M}\right)$. Hence we may assume that our global maximum $k$ satisfies $\xi_{1}(k)=$ $\lceil n-h-1\rceil$.

We can repeat this process to determine the location of all other " 1 " s in the binary expansion of our global maximum. For $2 \leq m \leq \delta$ we can define $\xi_{m}(k)=\max \left\{i<\xi_{m-1}(k): k_{i}=1\right\}$ and from (3.8) we conclude that if $\xi_{m}(k)<\lceil n-h+1-2 m\rceil$ and $k_{\xi_{m}}(k)+1=0$, we obtain a greater maximum by switching $k_{\xi_{m}(k)+1}$ and $k_{\xi_{m}(k)}$. Similarly, if $\xi_{m}(k) \geq$ $\lceil n-h+1-2 m\rceil+1$, then we can define $s_{m}(k)=\max \left\{i<\xi_{m}(k): k_{i}=0\right\}$ and define $k^{\prime}$ analogous to (3.9) to conclude that 


$$
\begin{aligned}
g\left(k^{\prime}\right)-g(k) & =-2^{s_{m}(k)-1}\left(n-h-s_{m}(k)-1-2 \sum_{i=s_{m}(k)+2}^{n} k_{i}\right) \\
& =-2^{s_{m}(k)-1}\left(n-h-s_{m}(k)-1-2\left(\xi_{m}(k)-s_{m}(k)-1+m-1\right)\right) \\
& =2^{s_{m}(k)-1}\left(2 \xi_{m}(k)-(n-h+1-2 m)-s_{m}(k)-2\right) \geq 0
\end{aligned}
$$

Thus, we can repeat (3.10) until we obtain a local maximum $k^{\#}$ of $g$ that satisfies $\xi_{m}\left(k^{\#}\right)=$ $\lceil n-h+1-2 m\rceil$. Note that for $m=\delta,\lceil n-h+1-2 m\rceil \in\{0,1\}$ and hence we set $\xi_{\delta}=1$ which agrees with our previous observation that we may take $k$ to be odd. Therefore, for $h<n-1$ (see Remark 1) the maximum of $g$ is attained at

$$
\begin{aligned}
k^{\star} & =\sum_{i=1}^{\delta-1} 2^{\xi_{i}-1}+1 \\
& =\sum_{i=1}^{\delta-1} 2^{\lceil n-h-2 i\rceil}+1
\end{aligned}
$$

In Remark 1 we consider the case $h \geq n-1$. Note also that if $n-2 \leq h<n-1$, then $\delta=1$ and (3.11) gives $k^{\star}=1$. Hence in this case we get $\Gamma^{\star}=n-h$. Therefore, for the remainder of this proof we will assume that $h<n-2$, and hence $\delta \geq 2$ and $k^{\star} \geq 3$. Let $\Xi_{1}=2^{\lceil n-h-2\rceil}$ and for $2 \leq i \leq \delta-1$ (whenever $\delta \geq 3$ ) let $\Xi_{i}=\Xi_{i-1}+2^{\lceil n-h-2 i\rceil}$. Then

$$
\sum_{i=1}^{k^{\star}-2} q(i)=\sum_{i=1}^{\Xi_{1}-1} q(i)+\sum_{m=2}^{\delta-1} \sum_{\Xi_{m-1}}^{\Xi_{m}-1} q(i)
$$

and by Lemma 3 we have that

$$
\sum_{i=1}^{\Xi_{1}-1} q(i)=\lceil n-h-2\rceil 2^{\lceil n-h-2\rceil-1}
$$

while

$$
\begin{aligned}
\sum_{i=\Xi_{m-1}}^{\Xi_{m}-1} q(i) & =\sum_{i=0}^{2^{\lceil n-h-2 m\rceil}-1}(q(i)+m-1) \\
& =\lceil n-h-2 m\rceil 2^{\lceil n-h-2 m\rceil-1}+2^{\lceil n-h-2 m\rceil}(m-1) \\
& =(\lceil n-h\rceil-2 m) 2^{\lceil n-h-2 m\rceil-1}+2^{\lceil n-h-2 m\rceil-1}(2 m-2) \\
& =(\lceil n-h\rceil-2) 2^{\lceil n-h-2 m\rceil-1}
\end{aligned}
$$

since for any $0 \leq i \leq 2^{\lceil n-h-2 m\rceil}, q\left(i+\Xi_{m-1}\right)=q(i)+m-1$. Thus

$$
\begin{aligned}
\sum_{i=1}^{k^{\star}-1} q(i) & =q\left(k^{\star}-1\right)+\sum_{i=1}^{k^{\star}-2} q(i) \\
& =(\delta-1)+\sum_{i=0}^{\Xi_{1}-1} q(i)+\sum_{m=2}^{\delta-1} \sum_{\Xi_{m-1}}^{\Xi_{m}-1} q(i) \\
& =(\delta-1)+(\lceil n-h\rceil-2) \sum_{m=1}^{\delta-1} 2^{\lceil n-h-2 m\rceil-1} .
\end{aligned}
$$


Hence we see that

$$
\begin{aligned}
g\left(k^{\star}\right) & =\left(1+\sum_{m=1}^{\delta-1} 2^{\lceil n-h-2 m\rceil}\right)(n-h)-2(\delta-1)-(\lceil n-h\rceil-2) \sum_{m=1}^{\delta-1} 2^{\lceil n-h-2 m\rceil} \\
& =(n-h-2 \delta+2)+(n-h-\lceil n-h\rceil+2) \sum_{m=1}^{\delta-1} 2^{\lceil n-h-2 m\rceil} \\
& =(n-h-2 \delta+2)+2^{\lceil n-h-2 \delta+2\rceil}(n-h-\lceil n-h\rceil+2)\left(4^{\delta-1}-1\right) / 3
\end{aligned}
$$

Finally, note that $g\left(k^{\star}\right)=\frac{1}{3}(2-h+\lfloor h\rfloor)\left(2^{2 \delta-1}+1\right)-1$ when $\lceil n-h\rceil$ is odd, and $g\left(k^{\star}\right)=\frac{1}{3}(2-h+\lfloor h\rfloor)\left(2^{2 \delta}-1\right)$ when $\lceil n-h\rceil$ is even, and both cases agree with $\Gamma^{\star}=n-h$ when $n-2 \leq h<n$. This completes the proof.

Remark 1 The above derivation was done under the assumption $\lceil n-h-1\rceil \geq 1$. Note that if $\lceil n-h-1\rceil=0$, then $\delta=1$ and it is immediate from (3.8) that the only " 1 " in the binary expansion of $k$ belongs to $k_{1}$. Therefore, in this special case $k^{\star}=1$ and $\Gamma^{\star}=n-h$ are the solutions to the above problem.

Proof of Theorem 4 In the proof of Theorem 5 we observed that if $n-2 \leq h<n$, the energy value $\mathcal{H}(\boxminus)+\Gamma^{\star}=\mathcal{H}(\boxminus)+n-h$ is first attained along the path $\left\{\gamma_{i}\right\}$ by the configuration $\gamma_{1}$. Furthermore, every optimal path attains this value in its initial step, and hence from the definition in (1.9) it is clear that $\mathscr{P}^{\star}=\{\boxminus\}$, and $\mathscr{C}^{\star}=\mathcal{M}\left(\gamma_{1}\right)$ is the set of all singleton configurations.

For $h<n-2$, it follows from the binary decomposition of $k^{\star}$ in (3.11) and the fact that $\gamma_{k^{\star}}$ is a good set, that $\gamma_{k^{\star}}$ is the configuration $\bigcup W_{i}$ given in the statement of Theorem 4. By Lemma 1, every optimal path $\gamma^{\prime}: \boxminus \rightarrow \boxplus$ must pass through $\mathcal{M}\left(\gamma_{k^{\star}}\right)$. Furthermore, from Lemma 5 it will follow that for any such path $\gamma^{\prime}$, if $\gamma_{i}^{\prime}$ is the first configuration along the path $\gamma^{\prime}$ that lies in $\mathcal{M}\left(\gamma_{k^{\star}}\right)$, then $\Phi\left(\gamma_{i-1}^{\prime}, \boxminus\right)<\Phi\left(\gamma_{i-1}^{\prime}, \boxplus\right)$. Hence by the third condition in (1.9) it follows that no configuration $\sigma$ with $|\sigma|<k^{\star}$ can be in $\mathscr{C}^{\star}$. Furthermore, no configuration $\sigma$ with $|\sigma|>k^{\star}$ satisfies " $\exists \xi \in \Omega$ such that $(\sigma, \xi) \in \mathcal{E}_{n}$ and $\Phi(\xi, \boxminus)<\Gamma^{\star}+\mathcal{H}(\boxminus)$ ". Hence, no configuration $\sigma$ with $|\sigma|>k^{\star}$ can have a neighbour in $\mathscr{P}^{\star}$, which by the first condition in (1.9) implies that $\sigma \notin \mathscr{C}^{\star}$. Lastly, if $|\sigma|=k^{\star}$ and $\sigma \notin \mathcal{M}\left(\gamma_{k^{\star}}\right)$, then by Lemma 1 we have that $\mathcal{H}(\sigma)>\mathcal{H}\left(\gamma_{k^{\star}}\right)$, and hence again $\sigma \notin \mathscr{C}^{\star}$. This shows that $\sigma \in \mathscr{C}^{\star}$ iff $\sigma \in \mathcal{M}\left(\gamma_{k^{\star}}\right)$, and thus completes the proof.

The validity of hypothesis (H2) in Theorem 3 is now trivial-all members of $\mathscr{C}^{\star}$ are the image of $\gamma_{k^{\star}}$ under different isomorphisms on $\mathcal{Q}_{n}$, hence they all have the same number of neighbours in $\mathscr{P}^{\star}$.

\section{Computation of the Prefactor $K$}

In this section we prove that the only way down from a critical configuration is through $\mathcal{M}\left(\gamma_{k^{\star}-1}\right) \cup \mathcal{M}\left(\gamma_{k^{\star}+1}\right)$ (Lemma 5). In Lemma 6 we calculate the cardinality of $\mathscr{C}^{\star}$, which we then use to prove Theorem 6.

The following variational equation (derived in Lemma 16.17 in [4]) gives an expression for $K$.

$$
1 / K=\min _{C_{1}, \ldots, C_{I}} \min _{f \in Q} \frac{1}{2} \sum_{\xi, \xi^{\prime} \in S^{\star}} \mathbb{1}_{\left\{\left(\xi, \xi^{\prime}\right) \in \mathcal{E}_{n}\right\}}\left[f(\xi)-f\left(\xi^{\prime}\right)\right]^{2}
$$


with

$$
Q=\left\{f: S^{\star} \rightarrow[0,1]: f_{\mid S_{\boxminus}}=1, f_{\mid S_{\boxplus}}=0 \text { and } f_{\mid S_{i}}=C_{i}\right\} .
$$

Here $\left\{S_{i}\right\}_{i=1}^{I}$ is a finite collection of all $S_{i} \subseteq \Omega$ that are mutually disjoint and satisfy

$$
\sigma \in \cup_{i} S_{i} \text { if and only if } \mathcal{H}(\sigma)<\Phi(\boxminus, \boxplus) \text { and } \Phi(\sigma, \boxminus)=\Phi(\sigma, \boxplus)=\Phi(\boxminus, \boxplus)
$$

The terms $C_{1}, \ldots, C_{I}$ are real numbers corresponding to the values that $f$ takes on $S_{1}, \ldots, S_{I}$. The set $S_{\boxminus}$ is defined by

$$
S_{\boxminus}=\{\sigma \in \Omega: \Phi(\sigma, \boxminus)<\Phi(\boxminus, \boxplus)\}
$$

and a similar definition is given to $S_{\boxplus}$. Lastly, $S^{\star} \subseteq \Omega$ is the set of all $\sigma \in \Omega$ such that $\Phi(\sigma, \boxminus) \leq \Phi(\boxminus, \boxplus)$ (and hence also $\Phi(\sigma, \boxplus) \leq \Phi(\boxminus, \boxplus)$ ).

We remark that for Lemma 16.17 in [4], $S^{\star}$ is defined to be the set of all $\xi \in \Omega$ with $\mathcal{H}(\xi) \leq \Phi(\boxminus, \boxplus)$. Let us denote that definition of $S^{\star}$ by $S_{a}^{\star}$, and note that it yields a bigger set since it may include $\xi \in \Omega$ that satisfy $\Phi(\xi, \boxminus)>\Phi(\boxminus, \boxplus)$. But any $\xi \in S_{a}^{\star} \backslash S^{\star}$ must lie in a component of the graph $\left(S_{a}^{\star}, \mathcal{E}_{n}\right)$ that is disconnected from $S_{\boxminus} \cup \mathscr{C}^{\star} \cup S_{\boxplus}$. Thus in (4.1) we may take $f \equiv 0$ on $S_{a}^{\star} \backslash S^{\star}$, which reduces the sum in (4.1) to a sum over the connected component of $\left(S_{a}^{\star}, \mathcal{E}_{n}\right)$ that contains the set $S_{\boxminus} \cup \mathscr{C}^{\star} \cup S_{\boxplus}$, which is precisely $\left(S^{\star}, \mathcal{E}_{n}\right)$. This justifies our definition of $S^{\star}$.

Recall from (1.11) that for any $\xi \in \Omega, \mathcal{M}(\xi)$ denotes the equivalence class of all $\sigma \in \Omega$ that are the image of $\xi$ under some graph isomorphism on $\mathcal{Q}_{n}$. To simplify (4.1), we will make use the following lemma which tells us that the only way down in energy from a critical configuration is through $\mathcal{M}\left(\gamma_{k^{\star}-1}\right)$ or $\mathcal{M}\left(\gamma_{k^{\star}+1}\right)$, where $k^{\star}$ is given in (3.11) and equal to the volume of a critical configuration.

Lemma 5 Suppose $0<h<n$ is not integer valued. Let $\xi \in \mathscr{C}^{\star}$ and $\sigma \in S^{\star}$ be neighbouring configurations, $(\xi, \sigma) \in \mathcal{E}_{n}$. Then $\sigma \in \mathcal{M}\left(\gamma_{k^{\star}-1}\right) \cup \mathcal{M}\left(\gamma_{k^{\star}+1}\right)$.

Proof Let $\sigma \in \Omega$ be such that $(\sigma, \xi) \in \mathcal{E}_{n}$, so that $|\sigma| \in\{|\xi|-1,|\xi|+1\}$. We will show that if $\sigma \notin \mathcal{M}\left(\gamma_{k^{\star}-1}\right) \cup \mathcal{M}\left(\gamma_{k^{\star}+1}\right)$, then $\mathcal{H}(\sigma)>\mathcal{H}\left(\gamma_{k^{\star}}\right)$ and hence $\sigma \notin S^{\star}$.

Let us first consider the case $n-2 \leq h<n$. By Theorem 4 , $\xi$ is a singleton configuration (i.e. $|\xi|=1$ ) and hence the only $\sigma \in \Omega$ satisfying $|\sigma|=|\xi|-1=0$ is $\sigma=\boxminus=\gamma_{0} \in \mathcal{M}\left(\gamma_{0}\right)$. Suppose now that $|\sigma|=|\xi|+1=2$, and assume w.l.o.g. that $\xi=\{\overline{0}\}$, where $\overline{0}$ is the vertex $(0, \ldots, 0) \in V_{n}$. Then if $\sigma \notin \mathcal{M}\left(\gamma_{2}\right)$, it must be that $\sigma=\{\overline{0}, w\}$ for some $w \in V_{n}$ that satisfies $(\overline{0}, w) \notin E_{n}$. This implies

$$
\mathcal{H}(\sigma)-\mathcal{H}(\boxminus)=2 n-2 h=2(\mathcal{H}(\xi)-\mathcal{H}(\boxminus))>\mathcal{H}(\xi)-\mathcal{H}(\boxminus)
$$

and hence $\sigma \notin S^{\star}$.

From here on we only consider $h<n-2$. Recall from the proof of Theorem 5 the definition $\delta=\lceil(n-h) / 2\rceil$. Again we may assume w.l.o.g. that $\xi=\gamma_{k^{\star}}=\bigcup W_{i}$ as defined in (1.12) and (1.13). If $|\sigma|=|\xi|+1, \sigma \notin \mathcal{M}\left(\gamma_{k^{\star}+1}\right)$, then $\sigma=\xi \cup\{w\}$ for some $w \notin W_{\delta-1}^{\prime}$ (see definition in (1.12) and (1.13)) or $w \in W_{\delta-1}^{\prime}$ and $\left(w, w_{\delta}\right) \notin E_{n}$, where $w_{\delta}$ is the unique vertex in $W_{\delta}$ (Note that since $\left|W_{\delta-1}\right| \in\{2,4\}$, this is only possible if $\left|W_{\delta-1}\right|=4$. See Figure 4). It follows that $w$ cannot have a neighbour in every $W_{i}$ for $1 \leq i \leq \delta$, since this is only possible for $w \in W_{\delta-1}^{\prime}$ with $\left(w, w_{\delta}\right) \in E_{n}$. Therefore, 


$$
\lceil n-h\rceil \text { odd }
$$

$$
\lceil n-h\rceil \text { even }
$$

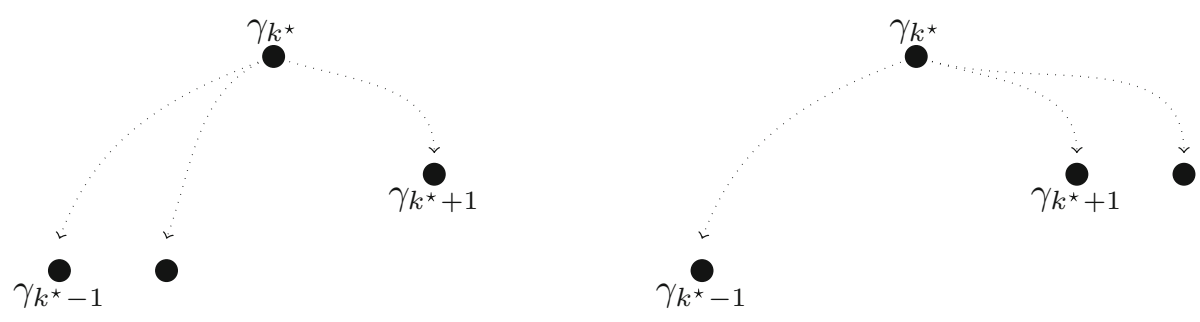

Fig. 4 When $\lceil n-h\rceil$ is odd, $\gamma_{k^{\star}}$ has two neighbours of volume $k^{\star}-1$ that have energy $\leq \Phi(\boxminus$, $\boxplus)$, and one neighbour of volume $k^{\star}-1$ that has energy $\leq \Phi(\boxminus, \boxplus)$. When $\lceil n-h\rceil$ is even, the opposite is true

$$
\begin{aligned}
\mathcal{H}(\sigma)-\mathcal{H}(\xi) & =-\left|\left\{(w, v) \in E_{n}: v \in \xi\right\}\right|+\left(n-\left|\left\{(w, v) \in E_{n}: v \in \xi\right\}\right|\right)-h \\
& \geq n-2(\lceil(n-h) / 2\rceil-1)-h \\
& \geq n-(n-\lfloor h\rfloor+1)+2-h \\
& >0
\end{aligned}
$$

and hence $\sigma \notin S^{\star}$.

Suppose now that $|\sigma|=|\xi|-1$ and $\sigma \notin \mathcal{M}\left(\gamma_{k^{\star}-1}\right)$. We will differentiate the case $\lceil n-h\rceil$ even from the case $\lceil n-h\rceil$ odd. Suppose first that $\lceil n-h\rceil$ is even, which in particular implies that $2 \delta=\lceil n-h\rceil$. Note that $\sigma=\xi \backslash\{w\}$ for some $w \in W_{i}$ for some $1 \leq i \leq \delta-1$, since if $w$ is the vertex in $W_{\delta}$, then by definition $\sigma=\gamma_{k^{\star}-1} \in \mathcal{M}\left(\gamma_{k^{\star}-1}\right)$, which is a contradiction. This implies

$$
\begin{aligned}
\mathcal{H}(\sigma)-\mathcal{H}(\xi) & =\left|\left\{(w, v) \in E_{n}: v \in \xi\right\}\right|-\left(n-\left|\left\{(w, v) \in E_{n}: v \in \xi\right\}\right|\right)+h \\
& =2\left|\left\{(w, v) \in E_{n}: v \in \xi\right\}\right|-n+h \\
& \geq 2(\lceil n-h-2 i\rceil+(i-1))-n+h \\
& =2\lceil n-h\rceil-2 i-2-n+h \\
& \geq 2\lceil n-h\rceil-2(\lceil(n-h) / 2\rceil-1)-2-n+h \\
& \geq\lceil n-h\rceil-n+h \\
& >0
\end{aligned}
$$

where the first inequality follows from the fact that if $w \in W_{i}$, then $w$ has $\lceil n-h-2 i\rceil$ neighbours in $W_{i}$ and one neighbour in each of $W_{i-1}, \ldots, W_{1}$. This shows again that $\sigma \notin S^{\star}$. Suppose now that $\lceil n-h\rceil$ is even, so that $2 \delta=\lceil n-h\rceil+1$. In this case $W_{\delta-1}$ is the set of vertices of a 1-dim sub-cube. As in the previous case, we have that $\sigma=\xi \backslash\{w\}$ for some $w \notin W_{\delta}$. Furthermore, we claim that if $w \in W_{\delta-1}$ then $\left(w, w_{\delta}\right) \in E_{n}$ where $w_{\delta} \in W_{\delta}$ is the unique vertex in that set. Indeed, if $\left(w, w_{\delta}\right) \notin E_{n}$ and $W_{\delta-1}=\left\{w, w^{\prime}\right\}$, then $\sigma=\xi \backslash\{w\}$ contains the set $W^{\$}=\left\{w^{\prime}, w_{\delta}\right\}$, which is the set of vertices of a 1-dim sub-cube that is adjacent to each $W_{i}, 1 \leq i \leq\lceil(n-h) / 2\rceil-2$ (see left-hand diagram in Figure 4). In other words, $\sigma \in \mathcal{M}\left(\gamma_{k^{\star}-1}\right)$, which is a contradiction. This implies

$$
\begin{aligned}
\mathcal{H}(\sigma)-\mathcal{H}(\xi) & \geq 2\left(\lceil n-h\rceil-i-1+\mathbb{1}_{\{i=\delta-1\}}\right)-n+h \\
& \geq 2\lceil n-h\rceil-2(\lceil(n-h) / 2\rceil-2)-2-n+h
\end{aligned}
$$




$$
\begin{aligned}
& \geq\lceil n-h\rceil+1-n+h \\
& >0
\end{aligned}
$$

and thus we conclude again that $\sigma \notin S^{\star}$. This completes the proof of the lemma.

An immediate conclusion from Lemma 5 is the following.

Corollary 1 Let $k^{\star}$ be as in (3.11). Then $\mathscr{P}^{\star}=\mathcal{M}\left(\gamma_{k^{\star}-1}\right)$.

In other words, $\mathscr{P}^{\star}$ is the set of all images of the set $\bigcup_{i=1}^{\delta-1} W_{i}$ given in (1.12) and (1.13), under isomorphisms on $\mathcal{Q}_{n}$ (see also Figure 1). We will now compute the cardinality of the critical set $\mathscr{C}^{\star}$. This quantity will be necessary for computing the prefactor $K$.

Lemma 6 Suppose $0<h<n$ and $h$ is not integer valued. Then $\left|\mathscr{C}^{\star}\right|=2^{n}$ for $h \geq n-2$, and

$$
\left|\mathscr{C}^{\star}\right|=\frac{n ! 2^{n}}{\lceil h\rceil !(2-\epsilon)}
$$

for $0<h<n-2$, where $\epsilon=\lceil n-h\rceil \bmod 2$.

Proof From Theorem 4 it follows immediately that if $h>n-2,\left|\mathscr{C}^{\star}\right|=\left|V_{n}\right|=2^{n}$. For $v \in V_{n}$ and $s \in \mathbb{N}, 1 \leq s \leq n$, let $\theta_{s}(v) \in V_{n}$ be the vertex that agrees with $v$ at every co-ordinate except at $s$. In other words, $\theta_{s}(v)_{i}=v_{i}$ for $i \neq s$, and $\theta_{s}(v)_{s}=1-v_{s}$. If $\mathcal{Q}_{r}$ is an $r$-dimensional sub-cube of $\mathcal{Q}_{n}(r<n)$, and $1 \leq s \leq n$ is such that $v_{s}=w_{s}$ for every $v, w \in \mathcal{Q}_{r}$ (in other words, the co-ordinate $s$ lies outside $\mathcal{Q}_{r}$ ), define $\theta_{s}\left(\mathcal{Q}_{r}\right)$ by

$$
\theta_{s}\left(\mathcal{Q}_{r}\right)=\left\{\theta_{s}(v): v \in \mathcal{Q}_{r}\right\}
$$

Note that $\theta_{s}\left(\mathcal{Q}_{r}\right)$ is also an $r$-dimensional sub-cube of $\mathcal{Q}_{n}$. We will say in this case that $s$ is an external co-ordinate of the sub-cube $\mathcal{Q}_{r}$. By Definition 1 and Theorem 4 , every configuration in $\mathscr{C}^{\star}$ can be constructed as follows. Start with any $\lceil n-h-2\rceil$-dimensional sub-cube $\mathcal{Q}_{1}$. There are $\left(\begin{array}{c}n \\ \lceil n-h-2\rceil\end{array}\right) \times 2^{n-\lceil n-h-2\rceil}$ different choices for such a sub-cube. Let $s_{1}$ be any external co-ordinate of $\mathcal{Q}_{1}$, and let $\mathcal{Q}_{2}$ be a $\lceil n-h-4\rceil$-dimensional sub-cube of $\theta_{s_{1}}\left(\mathcal{Q}_{1}\right)$. There are $(n-\lceil n-h-2\rceil) \times\left(\begin{array}{l}\lceil n-h-2\rceil \\ \lceil n-h-4\rceil\end{array}\right) \times 2^{2}$ ways to select $\mathcal{Q}_{2}$. Equation (3.11) implies that we should continue with this construction until we have chosen a $\lceil n-h-2 \delta+2\rceil$-dimensional sub-cube $\mathcal{Q}_{\delta-1}$ followed by a single vertex from the sub-cube $\theta_{s_{\delta-1}}\left(\mathcal{Q}_{s_{\delta-1}}\right)$, which will be identified with the 0 -dimensional sub-cube $\mathcal{Q}_{\delta}$. For $i \geq 2$, there are always two choices for the external co-ordinate $s_{i}$ of $\mathcal{Q}_{i}$, since both $\mathcal{Q}_{i}$ and $\theta_{s_{i}}\left(\mathcal{Q}_{i}\right)$ lie inside $\theta_{s_{i-1}}\left(\mathcal{Q}_{i-1}\right)$ (see Figure 1). Moreover there are $\left(\begin{array}{l}\lceil n-h-2 i\rceil \\ \lceil n-h-2 i-2\rceil\end{array}\right)$ ways to choose the co-ordinates of $\mathcal{Q}_{i+1}$, and $2^{2}$ ways to fix the two external co-ordinates of $\mathcal{Q}_{i+1}\left(\right.$ for $i+1<\delta$ ) that are in $\theta_{s_{i}}\left(\mathcal{Q}_{i}\right)$. Therefore, letting $b_{1}=(n-\lceil n-h-2\rceil)$ and $b_{i}=2$ for $2 \leq i \leq \delta-2$, we see that $\left|\mathscr{C}^{\star}\right|$ is given by 


$$
\begin{aligned}
\left|\mathscr{C}^{\star}\right|= & \left(\begin{array}{c}
n \\
\lceil n-h-2\rceil
\end{array}\right) \times 2^{n-\lceil n-h-2\rceil} \\
& \times\left[\begin{array}{c}
\delta-2 \\
\prod_{i=1} b_{i} \times\left(\begin{array}{c}
\lceil n-h-2 i\rceil \\
\lceil n-h-2 i-2\rceil
\end{array}\right) \times 2^{2}
\end{array}\right] \times 2 \times 2^{\lceil n-h-2 \delta+2\rceil} \\
= & 2^{3(\delta-2)+n-\lceil n-h-2\rceil+\lceil n-h-2 \delta+2\rceil}\left(\begin{array}{c}
n \\
\lceil n-h-2\rceil
\end{array}\right)\left[\prod_{i=1}^{\delta-2}\left(\begin{array}{c}
\lceil n-h-2 i\rceil \\
\lceil n-h-2 i-2\rceil
\end{array}\right)\right] b_{1} \\
= & \frac{n ! 2^{2(\delta-2)+n-\lceil n-h-2\rceil+\lceil n-h-2 \delta+2\rceil}(n-\lceil n-h-2\rceil)}{(n-\lceil n-h-2\rceil) !\lceil n-h-2 \delta+2\rceil} \\
= & \frac{n ! 2^{n}}{(n-\lceil n-h-2\rceil-1) !\lceil n-h-2 \delta+2\rceil}
\end{aligned}
$$

From this, the statement of the lemma follows.

We can now proceed with computing $K$.

Proof of Theorem 6 The proof works as follows. We first show that (4.1) can be simplified considerably. Following this simplification, it is necessary to count the neighbours (in $S_{\boxminus}$ and $S_{\boxplus}$ ) of any critical configuration. We do this by making use of Lemma 5.

Recall the definition of $k^{\star}$ in (3.11). It follows from equation (3.7) in the proof of Theorem 5 that if $h$ is not an integer, $g\left(k^{\star}\right)$ is a strict local maximum-i.e. $g\left(k^{\star}-1\right)<g\left(k^{\star}\right)$ and $g\left(k^{\star}\right)>g\left(k^{\star}+1\right)$. Furthermore, from equations (3.9) and (3.10) (in particular, the final inequality in both equations) it follows that $k^{\star}$ is the unique maximum of $g$. This in particular implies that $\gamma_{k^{\star}-1} \in S_{\boxminus}$ and $\gamma_{k^{\star}+1} \in S_{\boxplus}$.

From Lemma 5 we know that if $\sigma \in S^{\star},|\sigma|=k^{\star}-1$ and $(\sigma, \xi) \in \mathcal{E}_{n}$ for some $\xi \in \mathscr{C}^{\star}$, then $\sigma \in \mathcal{M}\left(\gamma_{k^{\star}-1}\right) \subseteq S_{\boxminus}$. Similarly, if $\sigma \in S^{\star},|\sigma|=k^{\star}+1$ and $(\sigma, \xi) \in \mathcal{E}_{n}$, then $\sigma \in \mathcal{M}\left(\gamma_{k^{\star}+1}\right) \subseteq S_{\boxplus}$. Indeed, every configuration $\sigma$ that is the neighbour of some $\xi \in \mathscr{C}^{\star}$ belongs to $S_{\boxminus} \cup S_{\boxplus}$. Furthermore, from Lemma 1 we know that if $\sigma \in \Omega,|\sigma|=k^{\star}$ and $\sigma \notin \mathscr{C}^{\star}$, then $\sigma \notin S^{\star}$.

We now claim that if $\sigma \in S^{\star},|\sigma|<k^{\star}$, then any minimizer $f$ in (4.1) satisfies $f(\sigma)=1$. Similarly, if $\sigma \in S^{\star},|\sigma|>k^{\star}$, then any minimizer $f$ in (4.1) satisfies $f(\sigma)=0$. Indeed, observe from (4.1) the obvious lower bound

$$
1 / K \geq \min _{f \in Q} \sum_{\xi \in \mathscr{C}^{\star}} \sum_{\xi^{\prime} \in S_{\boxminus} \cup S_{\boxplus}} \mathbb{1}_{\left\{\left(\xi, \xi^{\prime}\right) \in \mathcal{E}_{n}\right\}}\left[f(\xi)-f\left(\xi^{\prime}\right)\right]^{2} .
$$

Here we have dropped the constraint $f_{\mid S_{i}}=C_{i}$ since if $\xi \in \mathscr{C}^{\star}$ then $\xi \notin \bigcup_{i=1}^{I} S_{i}$, and similarly if $\xi^{\prime} \in S_{\boxminus} \cup S_{\boxplus}$, then $\xi^{\prime} \notin \bigcup_{i=1}^{I} S_{i}$. Hence if we only consider $f \in Q$ such that $f \equiv 1$ on $\left\{\sigma \in S^{\star}:|\sigma|<k^{\star}\right\}$ and $f \equiv 0$ on $\left\{\sigma \in S^{\star}:|\sigma|>k^{\star}\right\}$, then (4.5) becomes an equality, proving the claim.

Observe also that by symmetry, for every $\xi \in \mathscr{C}^{\star}$, the inner sum in the right-hand side of (4.5) is the same. Thus, taking any $\xi \in \mathscr{C}^{\star}$, we have that (4.1) has been reduced to

$$
\begin{aligned}
1 / K & =\left|\mathscr{C}^{\star}\right| \min _{f \in Q} \sum_{\xi^{\prime} \in S^{\star}} \mathbb{1}_{\left\{\left(\xi, \xi^{\prime}\right) \in \mathcal{E}_{n}\right\}}\left[f(\xi)-f\left(\xi^{\prime}\right)\right]^{2} \\
& =\left|\mathscr{C}^{\star}\right| \min _{f \in Q}\left(\sum_{\xi^{\prime} \in S_{\boxminus}} \mathbb{1}_{\left\{\left(\xi, \xi^{\prime}\right) \in \mathcal{E}_{n}\right\}}[f(\xi)-1]^{2}+\sum_{\xi^{\prime} \in S_{\boxplus}} \mathbb{1}_{\left\{\left(\xi, \xi^{\prime}\right) \in \mathcal{E}_{n}\right\}}[f(\xi)]^{2}\right)
\end{aligned}
$$


As pointed out in the proof of Lemma 5, if $h \geq n-2$ then the first sum in (4.6) contains only one term, namely $\xi^{\prime}=\boxminus$. It is also easy to see that in this case the second sum contains $n$ terms. Hence, for $n-2 \leq h<n$ we get

$$
1 / K=\left|\mathscr{C}^{\star}\right| \min _{f \in Q}\left([f(\xi)-1]^{2}+n[f(\xi)]^{2}\right) .
$$

For $h<n-2$, it was shown in Lemma 5 that if $\lceil n-h\rceil$ is even, there is a unique $\xi^{\prime} \in$ $\mathcal{M}\left(\gamma_{k^{\star}-1}\right)$ with $\left(\xi^{\prime}, \xi\right) \in \mathcal{E}_{n}$ (in particular, if $\xi=\bigcup_{i=1}^{\delta} W_{i}$ as in (1.12) and (1.13), then $\left.\xi^{\prime}=\bigcup_{i=1}^{\delta-1} W_{i}\right)$, while if $\lceil n-h\rceil$ is odd, there are two configurations $\xi^{\prime}, \xi^{\dagger} \in \mathcal{M}\left(\gamma_{k^{\star}-1}\right)$ that satisfy $\left(\xi^{\prime}, \xi\right) \in \mathcal{E}_{n}$ and $\left(\xi^{\dagger}, \xi\right) \in \mathcal{E}_{n}$ (i.e. $\xi^{\prime}=\bigcup_{i=1}^{\delta-1} W_{i}$ and $\xi^{\dagger}=W^{\$} \bigcup_{i=1}^{\delta-2} W_{i}$, where $W^{\$}$ was defined in the proof of Lemma 5). Similarly, if $\xi^{\prime} \in S_{\boxplus}$ and $\left(\xi^{\prime}, \xi\right) \in \mathcal{E}_{n}$ and $\xi=\bigcup_{i=1}^{\delta} W_{i}$, then $\xi^{\prime}=\xi \cup\{w\}$ where $w \in W_{\delta-1}^{\prime}$ is a vertex adjacent to the unique vertex $w_{\delta} \in W_{\delta}$-i.e. $\left(w, w_{\delta}\right) \in E_{n}$. If $\lceil n-h\rceil$ is odd then $W_{\delta-1}^{\prime}$ is a 1-dim sub-cube and there is a unique vertex $w$ that satisfies this. If $\lceil n-h\rceil$ is even, $W_{\delta-1}^{\prime}$ is a 2-dim sub-cube and there are two choices for $w$. See Figure 4.

Thus for $h<n-2$,

$$
1 / K=\left|\mathscr{C}^{\star}\right| \min _{f \in Q}\left((1+\epsilon)[f(\xi)-1]^{2}+(2-\epsilon)[f(\xi)]^{2}\right) .
$$

It is easy to see that the variational problems in (4.7) and (4.8) are solved by $f(\xi)=\frac{1}{n+1}$ and $f(\xi)=\frac{1+\epsilon}{3}$, respectively. Hence

$$
1 / K=\left|\mathscr{C}^{\star}\right| \frac{n}{n+1}
$$

when $n-2 \leq h<n$, and

$$
1 / K=\left|\mathscr{C}^{\star}\right| \frac{(1+\epsilon)(2-\epsilon)}{3}
$$

when $0<h<n-2$. Together with Lemma 6 , this completes the proof.

\section{Stability Levels and Reference Paths}

Theorem 3 states that hypotheses (H1) and (H2) hold whenever $0<h<n$. The latter was verified in Sect. 3, following the proof of Theorem 4. To verify (H1), we use a standard nucleation-path type of argument, similar to what is given in Chapter 17 in [4] for the Ising model in $\mathbb{Z}^{2}$. It exploits translation invariance in the underlying graph, and the possibility to initiate a uniformly optimal path (as defined in the statement of Lemma 2) starting from any vertex.

Proof of Theorem 3 Let $\sigma \in \Omega, \sigma \notin\{\boxminus, \boxplus\}$. We will show that $\mathscr{V}_{\sigma}<\Phi(\boxminus, \boxplus)=\mathscr{V}_{\boxminus}$, which by definition implies that $\sigma \notin \Omega_{m}$ and hence that the metastable states are given by $\Omega_{m}=\{\boxminus\}$.

Pick any $w \in \sigma$ s.t. $(w, y) \in E_{n}$ for some $y \in \bar{\sigma}$, and let $\gamma=\left(\gamma_{0}, \ldots, \gamma_{2^{n}}\right)$ be an optimal path with initial steps $\gamma_{1}=\{y\}$ and $\gamma_{2}=\{w, y\}$. This is always possible by translation invariance. We will show that the path $\left\{\sigma \cup \gamma_{i}\right\}_{i=0}^{S}$ (S will be defined below), going from $\sigma$ to $\sigma \cup \gamma_{S}$, satisfies $\mathcal{H}\left(\sigma \cup \gamma_{S}\right)<\mathcal{H}(\sigma)$, and $\mathcal{H}\left(\sigma \cup \gamma_{i}\right)-\mathcal{H}(\sigma)<\Phi(\boxminus$, $\boxplus)$ for all $0 \leq i \leq S$. By definition, this means that $\mathscr{V}_{\sigma}<\Phi(\boxminus, \boxplus)$, and hence $\sigma \notin \Omega_{m}$. Note first that

$$
\sigma \cap \gamma_{1}=\boxminus
$$


and

$$
1 \leq\left|\sigma \cap \gamma_{k}\right|<k \quad \forall k \geq 2
$$

Let us also denote by

$$
S=\min \left\{i \mid \mathcal{H}\left(\gamma_{i}\right) \leq \mathcal{H}(\boxminus)\right\}
$$

and note (by means of a simple computation) that $S \geq 2$ whenever $h<n$. Furthermore, for any $A, B \subseteq V_{n}$

$$
\begin{aligned}
|E(A \cup B, \overline{A \cup B})|+|E(A \cap B, \overline{A \cap B})| & \leq|E(A, \bar{A})|+|E(B, \bar{B})| \\
|A \cup B|+|A \cap B| & =|A|+|B| .
\end{aligned}
$$

It follows that for $2 \leq i \leq S$

$$
\begin{aligned}
\mathcal{H}\left(\sigma \cup \gamma_{i}\right)-\mathcal{H}(\sigma) & =\left(\left|E\left(\sigma \cup \gamma_{i}, \overline{\sigma \cup \gamma_{i}}\right)\right|-|E(\sigma, \bar{\sigma})|\right)-h\left(\left|\sigma \cup \gamma_{i}\right|-|\sigma|\right) \\
& \leq\left|E\left(\gamma_{i}, \overline{\gamma_{i}}\right)\right|-\left|E\left(\sigma \cap \gamma_{i}, \overline{\sigma \cap \gamma_{i}}\right)\right|-h\left(\left|\gamma_{i}\right|-\left|\sigma \cap \gamma_{i}\right|\right) \\
& =\mathcal{H}\left(\gamma_{i}\right)-\mathcal{H}\left(\gamma_{i} \cap \sigma\right) \\
& <\mathcal{H}\left(\gamma_{i}\right)-\mathcal{H}(\boxminus)
\end{aligned}
$$

where the last inequality follows from the fact that $\left|\gamma_{i} \cap \sigma\right|=m$ for some $m<i$. Hence, by uniform minimality of the configurations $\gamma_{j}$

$$
\mathcal{H}\left(\gamma_{i} \cap \sigma\right) \geq \mathcal{H}\left(\gamma_{m}\right)>\mathcal{H}(\boxminus) .
$$

Thus we have shown that the path $\left\{\sigma \cup \gamma_{i}\right\}_{i=0}^{S}$ satisfies $\mathcal{H}\left(\sigma \cup \gamma_{S}\right)<\mathcal{H}(\sigma)$, and $\mathcal{H}\left(\sigma \cup \gamma_{i}\right)-$ $\mathcal{H}(\sigma)<\Phi(\boxminus, \boxplus)$ for all $0 \leq i \leq S$, as required.

Finally, note that if $h \geq n$, then $\mathcal{H}\left(\gamma_{1}\right)-\mathcal{H}(\boxminus) \leq 0$ and from (5.1) it follows that (the derivation in (5.1) is also true for $i=1$, except that now the final inequality is not strict anymore) $\mathcal{H}\left(\sigma \cup \gamma_{1}\right)-\mathcal{H}(\sigma) \leq \mathcal{H}\left(\gamma_{1}\right)-\mathcal{H}(\boxminus) \leq 0$, thus $\sigma$ is not a local minimum of $\mathcal{H}$.

\section{Proof of Lemma 1}

In this section we will show that if $W$ is not a good set (as per Definition 1$),|E(W, \bar{W})|$ is not minimal-that is, $\exists U \subseteq V_{n},|U|=|W|$ such that $|E(U, \bar{U})|<|E(W, \bar{W})|$. Note that this is equivalent to showing $|E(W, W)|$ is not maximal. And unlike $|E(W, \bar{W})|$, the quantity $|E(W, W)|$ is invariant of the size of the cube in which $W$ is embedded, which will make it easier to work with.

We start with a definition. We will say that $W \subseteq V_{n}$ with $2^{r}<|W| \leq 2^{r+1}$ is wellcontained if there is a $(r+1)$-dimensional sub-cube of $\mathcal{Q}_{n}$ that contains $W$. Note that every set $W$ of size $|W|>2^{n-1}$ is well-contained, as is every good set (see Definition 1$)$. The following lemma shows that if $|E(W, \bar{W})|$ is minimal, then $W$ must be well-contained.

Lemma 7 If $W$ is not well-contained, $|E(W, W)|$ is not maximal.

Proof We begin with an observation. Let $\mathcal{C}_{0}$ be any sub-cube (of any dimension in $\{0,1, \ldots, n-1\}$ ), and let $\mathcal{C}_{1}=\theta_{s}\left(\mathcal{C}_{0}\right)$ for some external co-ordinate $s$ of $\mathcal{C}_{0}$ (recall from (4.3) that this means $\mathcal{C}_{0}$ and $\mathcal{C}_{1}$ are disjoint sub-cubes of the same size, and that there is some 
$1 \leq s \leq n$ such that every $u \in \mathcal{C}_{0}$ can be mapped to a $v \in \mathcal{C}_{1}$ by changing the value at $u_{s}$ ). If $W_{0} \subseteq \mathcal{C}_{0}, W_{1} \subseteq \mathcal{C}_{1}$ and $W=W_{0} \cup W_{1}$, then

$$
\begin{aligned}
|E(W, W)| & =\left|E\left(W_{0}, W_{0}\right)\right|+\left|E\left(W_{1}, W_{1}\right)\right|+\left|E\left(W_{1}, W_{0}\right)\right| \\
& \leq\left|E\left(W_{0}, W_{0}\right)\right|+\left|E\left(W_{1}, W_{1}\right)\right|+\min \left(\left|W_{1}\right|,\left|W_{0}\right|\right)
\end{aligned}
$$

where the inequality follows from the observation that every $v \in W_{1}$ has at most one neighbour in $W_{0}$, and vice versa. Furthermore,

claim If $W$ is a good set, then the inequality in (6.1) is an equality.

Proof of claim We will assume that $W_{0} \neq \varnothing$ and $W_{1} \neq \varnothing$, since otherwise the claim is trivially true. By the definition of a good set, there is some $l \in \mathbb{N}$, such that $W$ can be decomposed into $l$ disjoint good sets

$$
W=W^{1} \cup W^{2} \cdots \cup W^{l}, \quad l \in \mathbb{N} .
$$

Here $W^{i}, 1 \leq i \leq l$, is the set of all vertices in some $a_{i}$-dimensional sub-cube, with $a_{i}<a_{i-1}$. Furthermore, again from the definition of a good set, for every $i \geq 2$ we have $\bigcup_{j=i}^{l} W^{j} \subseteq$ $\theta_{b_{i-1}}\left(W^{i-1}\right)$ for some external co-ordinate $b_{i-1}$ of $W^{i-1}$ (this is analogous to the statement $\bigcup_{m=j+1} W_{m} \subseteq W_{j}^{\prime}$ in (1.13)). Then

$$
\left|E\left(W_{1}, W_{0}\right)\right|=\sum_{j, k}\left|E\left(W^{j} \cap \mathcal{C}_{0}, W^{k} \cap \mathcal{C}_{1}\right)\right| .
$$

Recall that $\mathcal{C}_{1}=\theta_{s}\left(\mathcal{C}_{0}\right)$, hence $s$ is an not an external co-ordinate of the $r+1$-dimensional sub-cube $\mathcal{C}_{0} \cup \mathcal{C}_{1}$. Note that for any $1 \leq i \leq l$, if $s$ is not an external co-ordinate of $W^{i},\left|W^{i} \cap \mathcal{C}_{0}\right|=\left|W^{i} \cap \mathcal{C}_{1}\right|=\frac{1}{2}\left|W^{i}\right|$ and this is also equal to $\left|E\left(W^{i} \cap \mathcal{C}_{0}, W^{i} \cap \mathcal{C}_{1}\right)\right|$. Thus, if $s$ is not an external co-ordinate of $W^{i}$ for all $1 \leq i \leq l$, then it must be that $\left|W_{0}\right|=\left|W_{1}\right|=\frac{1}{2} \sum\left|W^{j}\right|$ and

$$
\begin{aligned}
\left|E\left(W_{1}, W_{0}\right)\right| & =\sum_{j, k}\left|E\left(W^{j} \cap \mathcal{C}_{0}, W^{k} \cap \mathcal{C}_{1}\right)\right| \\
& =\sum_{j}\left|E\left(W^{j} \cap \mathcal{C}_{0}, W^{j} \cap \mathcal{C}_{1}\right)\right|=\frac{1}{2} \sum\left|W^{j}\right| .
\end{aligned}
$$

The second equality comes from the fact that if $j<k$, then any $v_{j} \in W^{j} \cap \mathcal{C}_{0}$ and any $v_{k} \in W^{k} \cap \mathcal{C}_{1}$ differ by at least two co-ordinates, namely $s$ and $b_{j}$ (since $W^{k} \subseteq \theta_{b_{j}}\left(W^{j}\right)$ ), and $s \neq b_{j}$.

Note that if $s$ is an external co-ordinate of $W^{i}$, then $s$ is an external co-ordinate of $W^{j}$ for all $j \geq i$. Let $\Xi=\min \left\{i: s\right.$ is an external co-ordinate of $\left.W^{i}\right\}$, and suppose w.l.o.g. that $W^{\Xi} \subseteq W_{0}$. Then $\left|W_{1}\right| \leq\left|W_{0}\right|$, and for any $i \in R=\left\{i>\Xi: W^{i} \subseteq W_{1}\right\}$, we have $W^{i} \subseteq \theta_{b \Xi}\left(W^{\Xi}\right)$ (see Figure 5). Hence for every $i \in R$ and $v \in W^{i}$, there is exactly one $w \in W_{0}$ (more precisely, $w \in W^{\Xi}$ ) such that $(v, w) \in E_{n}$. This shows that

$$
\left|E\left(W_{1}, W_{0}\right)\right|=\sum_{j=1}^{\Xi-1} \frac{1}{2}\left|E\left(W^{j} \cap \mathcal{C}_{0}, W^{j} \cap \mathcal{C}_{1}\right)\right|+\sum_{i \in R}\left|W^{i}\right|=\left|W_{1}\right|,
$$

and thereby proves the claim. 


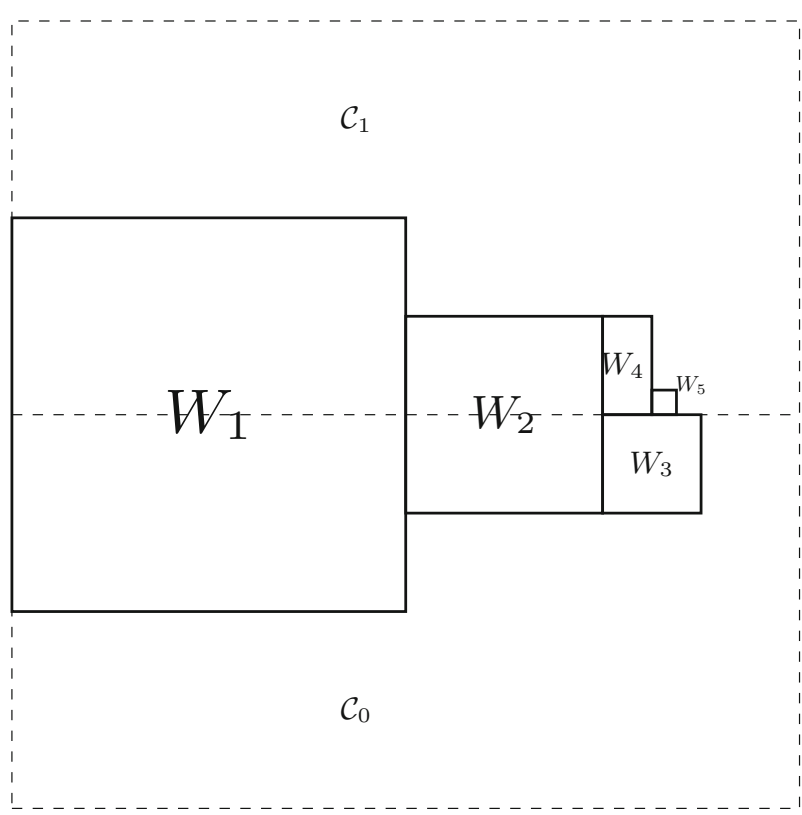

Fig. 5 Schematic representation of the decomposition $W^{1}, \ldots, W^{l}$, with $l=5$. Note that in this example $\Xi=3$ since $W^{3}$ is the largest sub-cube with $W^{3} \subseteq W_{0} \subseteq \mathcal{C}_{0}$. Every point in $W_{1}=\mathcal{C}_{1} \cap\left(\bigcup_{i=1}^{5} W^{i}\right)$ has a neighbour in $W_{0}$, which here corresponds to reflection about the dashed line in the middle

Let $r$ be such that $2^{r}<|W| \leq 2^{r+1}$. We may assume that $r+1 \leq n-1$, since if $2^{n-1}<|W|$ then $W$ is by definition well-contained in the cube $\mathcal{Q}_{n}$. We will start by induction on $n$. For $n=2$, the only sets that are not well-contained are $W^{1}=\{(0,0),(1,1)\}$ and $W^{2}=\{(1,0),(0,1)\}$. Clearly

$$
\left|E\left(W^{1}, W^{1}\right)\right|=\left|E\left(W^{2}, W^{2}\right)\right|=0
$$

is not maximal. Now suppose that the statement of the lemma is true whenever the setting is a hypercube of dimension less than or equal to $n-1$, and let $W \subseteq V_{n}$ be a set that is not wellcontained. Let $W_{0}=\left\{w \in W: w_{1}=0\right\}$ with $W_{1}$ defined similarly, so that $W_{0} \cup W_{1}=W$, and suppose w.l.o.g. that $\left|W_{0}\right| \geq\left|W_{1}\right|$. Note that the sets $W_{0}$ and $W_{1}$ are contained in two disjoint sub-cubes, call them $\mathcal{Q}_{n-1}^{0}$ and $\mathcal{Q}_{n-1}^{1}$, of dimension $n-1$.

Let $r_{0} \leq n-2$ be such that $2^{r_{0}}<\left|W_{0}\right| \leq 2^{r_{0}+1}$, and define $r_{1} \leq r_{0}$ in a similar manner. If $W_{0}$ is not well-contained, then by the inductive hypothesis $\left|E\left(W_{0}, W_{0}\right)\right|$ is not maximal. Hence we can find a good set $\widetilde{W}_{0}$ in $\mathcal{Q}_{n-1}^{0}$ with $\left|W_{0}\right|=\left|\widetilde{W}_{0}\right|$ and $\left|E\left(W_{0}, W_{0}\right)\right|<$ $\left|E\left(\widetilde{W}_{0}, \widetilde{W}_{0}\right)\right|$, and we can also replace $W_{1}$ by a good set $\widetilde{W}_{1}$ of the same size such that $\left|E\left(W_{1}, W_{1}\right)\right| \leq\left|E\left(\widetilde{W}_{1}, \widetilde{W}_{1}\right)\right|$. By $(6.1),\left|E\left(W_{0}, W_{1}\right)\right| \leq\left|W_{1}\right|$, and we may take $\widetilde{W}_{1}$ such that $\left|E\left(\widetilde{W}_{0}, \widetilde{W}_{1}\right)\right|=\left|\widetilde{W}_{1}\right|$ (by taking $\widetilde{W}_{1}$ to be a good set contained in $\theta_{1}\left(\widetilde{W}_{0}\right)$ ), hence it also follows that $\left|E\left(W_{0}, W_{1}\right)\right| \leq\left|E\left(\widetilde{W}_{0}, \widetilde{W}_{1}\right)\right|$. By (6.1) the set $\widetilde{W}=\widetilde{W}_{0} \cup \widetilde{W}_{1}$ satisfies $|E(W, W)|<|E(\widetilde{W}, \widetilde{W})|$, and hence $|E(W, W)|$ is not maximal. The same argument follows if $W_{1}$ is not well-contained. We may therefore assume that $W_{0}$ and $W_{1}$ are wellcontained.

Suppose first that $r_{0}+1<n-1$. Assuming $W_{0}$ and $W_{1}$ are well-contained, we can find two disjoint sub-cubes $\mathcal{Q}_{r_{0}+1}^{0}$ and $\mathcal{Q}_{r_{1}+1}^{1}$ containing $W_{0}$ and $W_{1}$ respectively (they are 

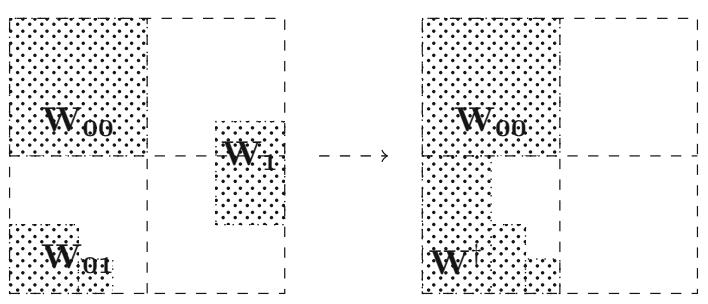

Fig. 6 The sets $W_{0}=W_{00} \cup W_{01}$ and $W_{1}$ are both well-contained, but their union $W=W_{0} \cup W_{1}$ is not. Replacing $W_{01} \cup W_{1}$ by $W^{\dagger}$, yields a new set $W^{\dagger} \cup W_{00}$ that is of the same size as $W$, but satisfies $\left|E\left(W^{\dagger} \cup W_{00}, W^{\dagger} \cup W_{00}\right)\right|>|E(W, W)|$

disjoint since every vertex in $W_{0}\left(W_{1}\right)$ has a $0(1)$ in its first co-ordinate, hence the same must be true for every vertex in $\mathcal{Q}_{r_{0}+1}^{0}\left(\mathcal{Q}_{r_{0}+1}^{1}\right)$ ). We may also assume that $W_{1} \subseteq \theta_{1}\left(W_{0}\right)$, since otherwise $\left|E\left(W_{0}, W_{1}\right)\right|<\min \left(\left|W_{0}\right|,\left|W_{1}\right|\right)$ and we can make the same argument as before to conclude that $|E(W, W)|$ is not maximal. Hence $W$ is contained in a $\left(r_{0}+2\right)$-dimensional sub-cube containing $\mathcal{Q}_{r_{0}+1}$ and $\mathcal{Q}_{r_{1}+1}$. Since $r_{0}+2 \leq n-1$, it follows from the inductive hypothesis that $|E(W, W)|$ is not maximal.

Finally, if $r_{0}+1=n-1$, we can decompose $W_{0}$ into $W_{0}=W_{00} \cup W_{01}$, with $W_{00}=$ $\left\{w \in W_{0}: w_{2}=0\right\}$ and with a similar definition for $W_{01}$. We can assume w.l.o.g. that $W_{0}$ and $W_{1}$ are good sets, since otherwise we can replace them by good sets $\widetilde{W}_{0}$ and $\widetilde{W}_{1}$ as was done in the previous case, to get $|E(W, W)| \leq|E(\widetilde{W}, \widetilde{W})|$, where $\widetilde{W}=\widetilde{W}_{0} \cup \widetilde{W}_{1}$. Then assuming $W_{0}$ is a good set, one of $W_{00}, W_{01}$ is the set of all vertices of a $(n-2)$-dimensional sub-cube. W.l.o.g. take this to be the set $W_{00}$, and note that $W_{01}$ is well-contained (since $W_{0}$ is a good set). Note that at least one of the inequalities $\left|E\left(W_{00}, W_{1}\right)\right| \leq \min \left(\left|W_{00}\right|,\left|W_{1}\right|\right)=\left|W_{1}\right|$ (since $\left.|W| \leq 2^{n-1}=\frac{1}{2}\left|W_{00}\right|\right)$ and $\left|E\left(W_{01}, W_{1}\right)\right| \leq \min \left(\left|W_{01}\right|,\left|W_{1}\right|\right)$ is strict, since each $w \in W_{1}$ has at most one neighbour in $W_{0}$, and that will be either in $W_{00}$ or $W_{01}$. Furthermore, we can find a good set $W^{\dagger}$ of same size as $\widehat{W}=W_{1} \cup W_{01}$ contained in the $(n-2)$-dimensional sub-cube that contains $W_{01}$ such that $\left|E\left(W^{\dagger}, W^{\dagger}\right)\right| \geq|E(\widehat{W}, \widehat{W})|$ and $\left|E\left(W^{\dagger}, W_{00}\right)\right|=$ $\left|W^{\dagger}\right| \geq\left|E\left(\widehat{W}, W_{00}\right)\right|$. But then at least one of the inequalities $\left|E\left(W^{\dagger}, W^{\dagger}\right)\right| \geq|E(\widehat{W}, \widehat{W})|$ and $\left|E\left(W^{\dagger}, W_{00}\right)\right| \geq\left|E\left(\widehat{W}, W_{00}\right)\right|$ is strict, and hence $\left|E\left(W^{\dagger} \cup W_{00}, W^{\dagger} \cup W_{00}\right)\right|>$ $|E(W, W)|$ (see Figure 6). This shows that $|E(W, W)|$ is not maximal, and completes the proof.

Proof of Lemma 1 As in the proof of Lemma 7, we will prove the statement of this lemma by induction on the size of the ambient hypercube. The case $n=2$ is simple, since the only sets that are not good are the two sets $W^{1}$ and $W^{2}$ from (6.3). Suppose now that whenever the setting is a hypercube of dimension less than or equal to $n-1, W$ is not a good set implies $|E(W, W)|$ is not maximal. Let $W$ be a subset of $\mathcal{Q}_{n}$ that is not good, $|W|=2^{r}+k$ for $1 \leq k \leq 2^{r}$ and $0 \leq r \leq n-1$. Then at least one of the following three statements is true:

(1) There is no $(r+1)$-dimensional sub-cube which contains the set $W$ (i.e. $W$ is not wellcontained).

(2) $\mathcal{Q}_{r+1}$ is a $(r+1)$-dimensional sub-cube of $\mathcal{Q}_{n}$ that contains $W$, and for any decomposition $\mathcal{Q}_{r+1}=\left(\mathcal{Q}_{r}^{0}, \mathcal{Q}_{r}^{1}\right)$ into two disjoint, $r$-dimensional sub-cubes, we have that $\bar{W} \cap \mathcal{Q}_{r}^{0} \neq \emptyset$ and $\bar{W} \cap \mathcal{Q}_{r}^{1} \neq \emptyset$.

(3) $\mathcal{Q}_{r+1}$ is a $(r+1)$-dimensional sub-cube of $\mathcal{Q}_{n}$ that contains $W$, and for any decomposition $\mathcal{Q}_{r+1}=\left(\mathcal{Q}_{r}^{0}, \mathcal{Q}_{r}^{1}\right)$ into two disjoint, $r$-dimensional sub-cubes, we have that $\bar{W} \cap \mathcal{Q}_{r}^{0}=\emptyset$ implies $W \cap \mathcal{Q}_{r}^{1}$ is not good. 
If the first statement is true, $|E(W, W)|$ is not maximal by Lemma 7. If the third statement is true, then the argument follows almost immediately from the inductive hypothesis. Indeed, if $W_{i}=W \cap \mathcal{Q}_{r}^{i}$ for $i \in\{0,1\}$, then replacing $W_{1}$ by a good set $\widetilde{W}_{1}$ of the same size and contained in $\mathcal{Q}_{r}^{0}$ implies that $\left|E\left(W_{1}, W_{1}\right)\right|<\left|E\left(\widetilde{W}_{1}, \widetilde{W}_{1}\right)\right|$ and $\left|E\left(W_{1}, W_{0}\right)\right| \leq\left|W_{1}\right|=\left|E\left(\widetilde{W}_{1}, W_{0}\right)\right|$. Suppose that the second statement is true. By the inductive hypothesis, if $r+1<n$ or if either one of $W_{0}, W_{1}$ is not good, $|E(W, W)|$ is not maximal. Hence we may assume that $r+1=n$. But now we can consider the set $U=\bar{W}$ instead, since $|E(U, \bar{U})|=|E(W, \bar{W})|$. Clearly $|U|<2^{n-1}$ and $U$ is not a good set ( $W$ satisfies the second statement above, so $U$ is not well-contained), hence again by the inductive hypothesis we have that $|E(U, U)|$ is not maximal (and hence $|E(U, \vec{U})|$ is not minimal). This proves that $|E(W, W)|$ is not maximal.

Acknowledgements This research is supported through NWO Gravitation Grant 024.002.003-NETWORKS. The author would like to thank Frank den Hollander and Siamak Taati for the helpful discussions and suggestions, as well as the referees for their comments and suggestions.

Open Access This article is distributed under the terms of the Creative Commons Attribution 4.0 International License (http://creativecommons.org/licenses/by/4.0/), which permits unrestricted use, distribution, and reproduction in any medium, provided you give appropriate credit to the original author(s) and the source, provide a link to the Creative Commons license, and indicate if changes were made.

\section{References}

1. Beltran, J., Landim, C.: Tunneling and metastability of continuous time markov chains. J. Stat. Phys. 140(6), 1065-1114 (2010)

2. Beltran, J., Landim, C.: Metastability of reversible finite state markov processes. Stoch. Process. Appl. 121, 1633-1677 (2011)

3. Beltran, J., Landim, C.: A martingale approach to metastability. Probab. Theory Relat. Fields 161(1), 267-307 (2015)

4. Bovier, A., den Hollander, F.: Metastability—A Potential-Theoretic Approach. Springer, Grundlehren der mathematischen Wissenschaften (2015)

5. Bovier, A., den Hollander, F., Spitoni, C.: Homogeneous nucleation for glauber and kawasaki dynamics in large volumes and low temperature. Ann. Probab. 38, 661-713 (2010)

6. Bovier, A., Eckhoff, M., Gayrard, V., Klein, M.: Metastability and small eigenvalues in markov chains. J. Phys. A 33, 447-451 (2000)

7. Bovier, A., Eckhoff, M., Gayrard, V., Klein, M.: Metastability in stochastic dynamics of disordered mean-field models. Probab. Theory Relat. Fields 119, 99-161 (2001)

8. Bovier, A., Eckhoff, M., Gayrard, V., Klein, M.: Metastability and low lying spectra in reversible markov chains. Commun. Math. Phys. 228, 219-255 (2002)

9. Bovier, A., Eckhoff, M., Gayrard, V., Klein, M.: Metastability in reversible diffusion processes. I. sharp asymptotics for capacities and exit times. J. Eur. Math. Soc. 6, 399-424 (2004)

10. Bovier, A., Manzo, F.: Metastability in glauber dynamics in the low temperature limit: beyond exponential asymptotics. J. Stat. Phys. 107, 757-779 (2002)

11. Cassandro, M., Galves, A., Olivieri, E., Vares, M.E.: Metastable behavior of stochastic dynamics: a pathwise approach. J. Stat. Phys. 35, 603-634 (1984)

12. Catoni, O., Cerf, R.: The exit path of a markov chain with rare transitions. ESAIM Probab. Stat. 1, 95-144 (1997)

13. Cerf, R., Manzo, F.: Nucleation and growth for the ising model in dimensions at very low temperatures. Ann. Probab. 41, 3697-3785 (2013)

14. Dehghanpour, P., Schonmann, R.H.: Metropolis dynamics relaxation via nucleation and growth. Commun. Math. Phys. 188, 89-119 (1997)

15. Dommers, S.: Metastability of the ising model on random regular graphs at zero temperature. Probab. Theory Relat. Fields (2015). doi:10.1007/s00440-015-0682-0

16. Dommers, S., den Hollander, F., Jovanovski, O., Nardi, F.: Metastability for glauber dynamics on random graphs. Ann. Appl. Probab. (2016) (to appear)

17. Hart, S.: A note on edges of n-cube. Discret. Math. 14, 157-163 (1976) 
18. Kotecky, R., Olivieri, E.: Stochastic models for nucleation and crystal growth. In: Probabilistic Methods in Mathematical Physics, pp. 264-275. World Scientific, Riverside (1991)

19. Kotecky, R., Olivieri, E.: Droplet dynamics for asymmetric lsing model. J. Stat. Phys. 70(5/6) (1993)

20. Kotecky, R., Olivieri, E.: Shapes of growing droplets-a model of escape from the metastable phase. J. Stat. Phys. 75, 409-506 (1994)

21. Neves, E.J., Schonmann, R.H.: Critical droplets and metastability for a glauber dynamics at very low temperature. Commun. Math. Phys. 137, 209-230 (1991)

22. Olivieri, E., Scoppola, E.: Markov chains with exponentially small transition probabilities: first exit problem from general domain i. the reversible case. J. Stat. Phys. 79, 613-647 (1995)

23. Olivieri, E., Scoppola, E.: Markov chains with exponentially small transition probabilities: first exit problem from general domain ii. the general case. J. Stat. Phys. 84, 987-1041 (1996)

24. Olivieri, E., Vares, M.: Large deviations and metastability (Encyclopedia of Mathematics and its Applications-Book 100). Cambridge University Press, Cambridge (2005) 